\section{Simulation of Oleuropein Structural Conformation in Vacuum, Water and Triolein-water systems using Molecular Dynamics}

\author{
$8{ }^{a}$ Food Engineering Division, National Food Research Institute, NARO, Tsukuba, 2-1-12 \\ 9 Kannondai, Tsukuba, Ibaraki 305-8642, Japan. \\ Safa Souilem ${ }^{\mathrm{a}, \mathrm{b}, \mathrm{c}}$,Witcha Treesuwan ${ }^{\mathrm{d}}$, Isao Kobayashi ${ }^{\mathrm{a}, \mathrm{b}}$, Nauman Khalid ${ }^{\mathrm{a}, \mathrm{b}}$, \\ Zouhaier Bouallagui ${ }^{\mathrm{c}}$, Marcos A. Neves ${ }^{\mathrm{a}, \mathrm{b}}$, Kunihiko Uemura ${ }^{\mathrm{a}}$, \\ Hiroko Isoda ${ }^{\mathrm{b}}$, Sami Sayadic, Mitsutoshi Nakajima ${ }^{\mathrm{a}, \mathrm{b} * *}$ \\ ${ }^{b}$ Alliance for Research on North Africa (ARENA), University of Tsukuba, Tsukuba,1-1-1 \\ Tennoudai, Tsukuba, Ibaraki 305-8572, Japan. \\ ${ }^{c}$ Center of Biotechnology of Sfax (CBS), B.P. 1177, Sfax 3018, Tunisia. \\ ${ }^{d}$ Institute of Food Research and Product Development, Kasetsart University, Bangkok 10900, \\ Thailand \\ * Corresponding author. Tel.: +8129838 8025; fax: +81 298388122. \\ E-mail address:isaok@affrc.go.jp(I. Kobayashi) \\ ** Corresponding author. Tel.: +81 29853 4703; fax: +81 298534703 \\ E-mail address:nakajima.m.fu@u.tsukuba.ac.jp (M. Nakajima)
}

Keywords: oleuropein, MD, conformation, torsion, RDF, energy, interface 


\section{Abstract}

22 Oleuropein, the main phenolic compound of olive leaves, exhibits a unique blend of

23 biological activities and has been shown to locate itself at the oil-water $(\mathrm{O} / \mathrm{W})$ interface.

24 This behavior could influence on the physico-chemical properties of dispersed systems

25 such as emulsions. In this work, we study the effect of the microenvironment (vacuum,

26 water, and triolein-water) on the conformational preferences of oleuropein using

27 molecular dynamics (MD) simulations at $300 \mathrm{~K}$ for at least $30 \mathrm{~ns}$. The seven torsions

28 that describe the flexible skeleton of oleuropein were monitored together with the

29 distance between the glucose $(\mathrm{Glu})$ and hydroxytyrosol (Hyd) moieties $\left(\mathrm{d}_{\mathrm{glu}-\mathrm{hyd}}\right)$ of the

30 molecule. The obtained trajectories demonstrated that oleuropein adopts different

31 conformations that depend on the environment. The preferential conformers in each

32 system were analyzed for their molecular geometry and internal energy. In vacuum, the

33 oleuropein preferential conformation is tight with the glucose moiety in close proximity

34 with the hydroxytyrosol moiety. In water, oleuropein preferential conformers presented

35 large differences in their structural properties, varying from a close like $U$ form, and a

36 semi-opened form, to an opened form characterized by high fluctuations in $\mathrm{d}_{\text {glu-hyd }}$

37 values. In a triolein-water system; oleuropein tends to adopt a more open form where

38 the glucose moiety could be approximately aligned with the hydroxytyrosol and elenolic

39 acid moieties. Based on a calculation at the HF/6-31G* level, these flexibilities of

40 oleuropein required energy of $19.14 \mathrm{kcal} / \mathrm{mol}$ in order to adapt the conformation

41 between water and triolein-water system. A radial distribution function (RDF) analysis

42 showed that specific hydroxyl groups of Hyd and Glu interact with water molecules,

43 enabling us to understand the amphiphilic character of oleuropein at the triolein-water 
interface. MD calculations together with interfacial tension measurements revealed the oleuropein binding at $\mathrm{O} / \mathrm{W}$ interface is an enthalpy driven mechanism.

\section{Introduction}

Major and minor biomolecules from olive plants and its processing byproducts have been consumed for a long time in the Mediterranean diet without an understanding of their structure, function, and interactions within the olive fruit or in the human body

51 (Uccella, 2000). Advanced scientific research has revealed that a Mediterranean diet rich in olive oil is associated with a decrease in risk for cardiovascular disease, obesity, and diabetes (López-Miranda et al., 2010). These protective functions may originate

54 from the lipid components of such a diet as well as phytochemicals including polyphenols, which occurr in olive oil and olive plants. Among protective polyphenols, oleuropein, which is the main component of bitterness in olives, is well known to exhibit a unique blend of biological activities (Bulotta et al., 2013; Cicerale, Lucas, \& Keast, 2010). Oleuropein is a secondary metabolite synthesized via a branching in the melalonic acid during the formation of oleosides (Omar, 2010). Oleuropein can be extracted from the leaves and drupes of the olive tree (Olea europaea L.), especially

61 present in the leaves (60-90 mg/g dry weight) (Ghanbari, Anwar, Alkharfy, Gilani, \& 62 Saari, 2012). Purified oleuropein and its aglycon forms were characterized 63 spectroscopically using the NMR and FABS-MS methods (Capasso et al., 1997).

64 The oleuropein structure depicted in Fig.1, shows that this polar glycoside includes

65 three compartments, a glucose molecule, elenolic acid and hydroxytyrosol. During fruit

66 maturation or industrial processing of extra virgin olive oil, enzymatic hydrolysis by 
$\beta$-glucosidase results in cleavage of the glucose moiety from the non-polar oleuropein aglycone, and the hydrolysis action of esterase engenders the liberation of hydroxytyrosol and elenolic acid (Gutierrez-Rosales, Romero, Casanovas, Motilva, \& Mínguez-Mosquera, 2010). Oleuropein provides a natural defense against pathogenic attack and plays a role in structuring the cell-wall skeleton by acting as a cross- linker between the different polysaccharide moieties in the plant cells (Uccella, 2000).

The activity of polyphenolic compounds depends on their structural characteristics (Leopoldini, Russo, \& Toscano, 2011). Molecular conformation can be investigated using a multitude of scientific techniques including X-ray crystallography, NMR spectroscopy, and computational modeling. A structural clarification of oleuropein in a human biological environment would be of great interest since it helps us to understand the mechanisms of its biological activities as correlated with its physico-chemical properties. In this field, numerous works have focused on the conformation of the oleuropein binding to some key proteins involved in the pathway of oleuropein mediated diseases using molecular modeling (MD). MD studies indicate that oleuropein binds to the peroxisome proliferator activating receptors involved in atherosclerosis expression (Andreadou et al., 2006; Huang, Huang, \& Lee-Huang, 2010). Oleuropein conformation was strongly dependent on the receptor type $\alpha, \beta$, or $\gamma$ (Huang et al., 2010). Lee-Huang et al. (2007) investigated conformation of the oleuropein binding to the retrovirus (HIV-1) envelop glycoprotein (gp41) using molecular docking and MD simulations. The conformation of isolated oleuropein in vacuum has shown a closed structure in which its phenolic hydrogen forms a hydrogen-bond network with the hydroxyl groups of the glucose moiety(Gikas, Bazoti, \& Tsarbopoulos, 2007). However, the MD simulations of oleuropein in vacuum did not consider the chemical reactions 
91 that are carried out in solution, where this compound would really exist. Thus, a study 92 of a possible solvent effect on oleuropein conformation should be considered. For 93 instane, an understanding of oleuropein conformation within the olive cellular 94 environments will be very important for interpreting its histological distribution, and 95 investigating its reactivity with enzymes and other physiological partners.

96 Recently, several biological activities of oleuropein have been correlated to its 97 surface activity (Di Mattia, Sacchetti, \& Pittia, 2011). For instance, the antimicrobial activity of oleuropein can be attributed to its ability to damage the bacterial membrane

99 (Bisignano et al. 1999). A few research groups suggested that the effectiveness of 100 oleuropein, as an antioxidant in a membrane of phospholipid bilayer, is associated with 101 the membrane surface rather than penetration of oleuropein into the membrane 102 (López-Miranda et al. 2010; Caturla, Pérez-Fons, Estepa, \& Micol, 2005).

103 From a physical point of view, the surface activity of oleuropein would be important 104 during extraction of the olive oil, which involves several unit operations of biphasic 105 systems: mainly malaxation and centrifugation (Di Giovacchino, Costantini, Ferrante, \& 106 Serraiocco, 2002). Our previous study reveals that oleuropein was able to significantly 107 reduce the interfacial tension of triglyceride oil-water interfaces (Souilem et al. 2014), 108 which confirms its high surface activity. We also proved that oleuropein is the major 109 surface active compound in olive leaf extract (OLE). Ghahfarokhi et al. (2015) 110 suggested the use of OLE as a naturally occurring surfactant since it was able to reduce 111 the interfacial tension between kerosene and water from 36.5 to $14 \mathrm{mN} / \mathrm{m}$. Souilem et al. 112 (2014) used microchannel emulsification to demonstrate the ability of oleuropein to 113 stabilize oil-in-water $(\mathrm{O} / \mathrm{W})$ emulsions. Although this experimental evidence about the 
$114 \mathrm{O} / \mathrm{W}$ interfacial activity of oleuropein, there is no available explanation at molecular

115 scale, on the behavior of oleuropein at $\mathrm{O} / \mathrm{W}$ interface. An investigation on the change

116 of oleuropein conformation upon adsorption to the $\mathrm{O} / \mathrm{W}$ interface could lead to a better

117 understanding of its amphiphilic character.

118 In this paper we extend our earlier study by focusing on the active conformation of

119 oleuropein at the $\mathrm{O} / \mathrm{W}$ interface and its conformational change according to its nature 120 and the media (vacuum, water, and triolein-water systems) using MD simulations.

122

\section{Computational methods}

\section{2.1. Setup model and simulation details}

124 The initial structures of oleuropein and triolein were generated using molecular 125 modeling software (ChemDraw). To overcome the high flexibility of the molecule, a 126 conformational search had been done to obtain the most stable torsional angle of the 127 molecule. The configuration at the lowest energy was selected to be further subjected to 128 a full geometry optimization at the force field B3LYP/6-31G $(\mathrm{d}, \mathrm{p})$ by the application of 129 Gaussian 09.

130 The topology of oleuropein was generated through the Dundee PRODRG2 Server. The 131 atomic charge of oleuropein was obtained from the optimized structure at the 132 B3LYP/6-31G (d,p) level of theory. Bond interactions were calculated based on the 133 GROMOS96 force field. A united atom force field (http://wcm.ucalgary.ca/tieleman) 134 based on lipid parameters (Tieleman and al. 2006) were applied to triolein. The 135 extended simple point charge (SPC/E) model of water was used to describe the solvent 
water. MD simulations were carried out in triplicate using the GROMACS 4.6.1

137 software package. The systems for oleuropein in vacuum, water and triolein-water were 138 setup as follows.

\section{2.1.1. Vacuum system}

140 Oleuropein was minimized without any constraints before 50ns. MD simulations at 300

141 K. The non-bonded interactions were settled with a cutoff of $0.7 \mathrm{~nm}$.

\section{$142 \quad$ 2.1.2. Water system}

143 A single oleuropein molecule was placed in the center of a cubic box $5 \mathrm{~nm}$ inlength.

144 The total number of water molecules was 4115 . The system was first subjected to an

145 initial minimization for $100 \mathrm{ps}$, followed by equilibration in a canonical ensemble

146 (NVT) for $200 \mathrm{ps}$ and an isothermal-isobaric ensemble (NPT) for $200 \mathrm{ps}$ and $400 \mathrm{ps,}$

147 respectively, under periodic boundary conditions and with a time step of 2 fs. The

148 temperature was set to $300 \mathrm{~K}$ using a Parrinello-Rahman thermostat with a coupling

149 constant of 0.1 ps. The total pressure was isotropically kept at 1 atm using a

150 Parrinello-Rahman pressures coupling of 0.5 ps. Electrostatic interactions were modeled

151 with the particle mesh Ewald (PME) approach using a grid spacing of $0.16 \mathrm{~nm}$. The MD

152 simulation was subsequently conducted for $50 \mathrm{~ns}$ at constant temperature of $300 \mathrm{~K}$

153 under the NPT ensemble as previously set conditions.

\section{$154 \quad$ 2.1.3. Triolein-water system}

155 A box of triglyceride phase composed of 199 triolein molecules was generated, 156 minimized and equilibrated by GROMACS. A oleuropein-triolein-water system was 157 then built from three layers of water, triolein and water as illustrated in Fig. 2.b. The 
dimensions of the resulting box were $6.82 \mathrm{~nm}$ X $6.82 \mathrm{~nm}$ X $20.62 \mathrm{~nm}$. The oleuropein

159 phase having a thickness of $6.92 \mathrm{~nm}$, was intercalated between two opposing cubic

160 water boxes aligned along the z-axis. Each water box has a thickness of $6.85 \mathrm{~nm}$ and

16121271 molecules. Subsequently, one oleuropein molecule was added at each

162 triolein/water interface. Three sets of simulations with different starting orientation of

163 oleuropein were conducted (Fig.2.b). During the first simulation (S1), oleuropein was

164 placed where its glucose moiety was orientated into the triolein phase. In the second

165 simulation (S2), oleuropein was placed such that its glucose moiety was oriented into

166 the water phase. The final simulation (S3) was carried with oleuropein in which the

167 glucose and hydroxytyrosol moieties had been aligned parallel to the interface.

168 Initially, the system was subjected to energy minimization using the Steepest Descent

169 and Conjugated gradient methods to remove the initial strain. Equilibration was carried

170 out under canonical ensemble for $100 \mathrm{ps}$. The temperature was raised to $300 \mathrm{~K}$ and held

171 constant using a Parrinello-Rahman thermostat with a coupling constant of 0.1 ps.

172 Electrostatic interactions were modeled using the particle mesh Ewald (PME) approach

173 with a grid spacing of $0.16 \mathrm{~nm}$ and an interpolation order (PME order of 4). Van der

174 Waals interactions were computed with a cut-off of $1.4 \mathrm{~nm}$. All bonds involving

175 hydrogen atoms were constrained using the LINCS algorithm. The integration time step

176 was 2 fs. The density of the system was equilibrated in the isothermal-isobaric (NPT)

177 ensemble for 200 ps. The total pressure was held at 1 atm using a Parrinello-Rahman

178 pressure coupling of 0.5 ps. Production simulations were carried out for $30 \mathrm{~ns}$ at a

179 constant temperature of $300 \mathrm{~K}$ under the NPT ensemble as previously set conditions. To

180 maintain a stable interface, semi-isotropically pressure coupling was applied allowing

181 the length of the box in $\mathrm{z}$ direction to change but holding the box lengths in the $\mathrm{x}$ and $\mathrm{y}$ 
182 directions fixed to their initial values. Such an MD setup has previously been

183 demonstrated to be optimal for simulating the equilibrium properties of a

184 triglyceride-water interface (Henneré et al. 2009).

185 2.2. Analysis of MD trajectories

$186 \quad$ 2.2.1. Torsions analyses

187 The conformation of oleuropein was analyzed by selecting of the seven important 188 torsions $\left(\mathrm{T}_{1}, \mathrm{~T}_{2}, \mathrm{~T}_{3}, \mathrm{~T}_{4}, \mathrm{~T}_{5}, \mathrm{~T}_{6}\right.$ and $\left.\mathrm{T}_{7}\right)$ defined in Fig.2.a.These torsions belong to the 189 backbone skeleton of oleuropein and describe the possible rotational degrees of freedom 190 along the three successive chemical-bond vectors between elenolic acid and glucose.

191 The torsions were preset to vary within the range $-180^{\circ}$ to $+180^{\circ}$. Their distributions 192 were analyzed from the frames, after reaching thermodynamic equilibrium. 193 Representative conformers of oleuropein in each environment were chosen according to 194 the highest population of the determined torsional angles.

\section{$195 \quad 2.2 .2$ Energy calculation}

196 To complement the structural analysis, potential energy calculations were 197 performed for the selected preferable snapshots using the Gaussian09 program 198 package. Hydrogen and heavy atoms in each oleuropein conformer were optimized at $199 \mathrm{HF} / 3-21 \mathrm{G}$ and the energy was obtained at $\mathrm{HF} / 6-31 \mathrm{G} *$ levels.

\subsubsection{Distance analysis}

201 To approximate the spatial flexibility of oleuropein in the different studied systems; the

202 end-to-end distance of the molecule $\left(\mathrm{d}_{\mathrm{glu}-\mathrm{hyd}}\right)$ was measured during the simulations. 
$203 \mathrm{~d}_{\text {glu-hyd }}$ represents the distance between the centers of the hydroxytyrosol and glucose 204 moieties.

205 2.2.4. Radial distribution function $(R D F)$ analyses

206 The RDF analysis aimed to investigate the hydrogen bonding betweenfunctional groups 207 of oleuropein (hydroxyl and ester) and environmental water molecules. Therefore, pair 208 radial distributions were analyzed between the oxygen atoms in the oleuropein molecule 209 (O2, O3, O4, O5, O6, OAR, OAV, OAW, OBE and OBA) and the oxygen atoms of 210 water molecules. The distance of the $1^{\text {st }}$ hydration shell and the numbers of water 211 molecules were obtained for both the water and the triolein/water system. The 212 heteroatomic distances within $3.00 \AA$ were considered for the hydrogen bonding 213 interactions.

214 2.2.5. Determination of the standard enthalpy of oleuropein adsorption

215 2.2.5.1. MD calculations

216 Adsorption enthalpy of oleuropein in triolein-water system was obtained from the last 21715 ns of production trajectories using molecular mechanics Poisson-Boltzmann surface 218 area (MM-PBSA) approach implemented in g_mmpbsa (Rashmi et al. 2014). Briefly, 219 binding enthalpies between oleuropein and triolein in gas phase were combined with the 220 polar salvation and nonpolar salvation energy according to the thermodynamic cycle. 221 Implicit salvation model was applied using dielectric constant of 80 with grid space of

$2221.5 \AA$. Solvent probe radius was $1.4 \AA$. In total, 1000 snapshot structures were used in 223 the MM-PBSA calculations and analysis. 
225 The standard enthalpy of oleuropein adsorption at soybean oil/water interface was 226 determined based on interfacial tension measurements following the method described 227 by (Krassimir and Kralchevsky. 2012).

228 Interfacial tension measurements were carried out using a pendant drop tensiometer 229 analyzer (PD-W, Kyowa Interface Science Co., Ltd., Saitama, Japan); following the 230 method by (Souilem et al. 2014). Measurements were carried out using aqueous 231 solutions of highly purified oleuropein (purity 93\%) (Extrasynthèse, France) and refined 232 soybean oil (Wako Pure Chemical Industries, Ltd. (Osaka, Japan)).

233 First, the standard free energy of oleuropein adsorption $\Delta \mathrm{G}$ was determined by linear 234 fits of interfacial tension isotherms at different temperatures $\left(15^{\circ} \mathrm{C}, 25^{\circ} \mathrm{C}, 35^{\circ} \mathrm{C}\right.$ and $23545^{\circ} \mathrm{C}$ ) with the help of the theoretical model of adsorption defined by Eq. (1):

$$
K \ln \left(\frac{C}{W}\right)=\frac{1}{\Gamma \mathrm{d}} \pi \mathrm{s}+\Delta G
$$

237 Where $\Gamma_{\mathrm{d}}$ (const) is the adsorption layer of oleuropein, $\omega=\rho_{\mathrm{w}} / 18$ is the molar 238 concentration of water; $\rho_{\mathrm{w}}(\mathrm{g} / \mathrm{L})$ is the mass density of water and $\pi_{\mathrm{s}}(\mathrm{mN} / \mathrm{m})$ is the 239 surface pressure. $\Delta \mathrm{G}$ is determined from the intercept of the linear regression of plots 240 from experimental data for $\pi_{\mathrm{s}}(\mathrm{C})$ as $K T \ln \left(\frac{C}{W}\right)$ in accordance with Eq. (1). The Gibbs 241 free energy $(\Delta \mathrm{G})$ of an isothermal transfer of surfactant molecules from bulk solution to 242 interface is defined by Eq. (2):

$$
\Delta G=\Delta H-T \Delta S
$$

244 Where $\Delta \mathrm{H}(\mathrm{KJ} / \mathrm{mol})$ is the free enthalpy of adsorption and $\Delta \mathrm{S}(\mathrm{KJ} / \mathrm{mol})$ is the free 245 entropy of adsorption. $\Delta \mathrm{H}$ is determined from the intercept of the linear regression of 246 plots from the obtained experimental $\Delta \mathrm{G}$ as $\mathrm{T}^{\circ}$ in accordance with Eq. (2). 


\section{3. Results and discussion}

\section{3.1. Equilibrium profile of the system}

250 Analyses were performed after the system shifted towards equilibrium. Equilibrium of 251 the system was monitored through the root mean squared deviation (RMSD) and 252 density. RMSD values of oleuropein reached equilibrium after 200 ps for vacuum, 2 ns 253 for water and $15 \mathrm{~ns}$ for triolein-water systems.

254 The density profiles of water and triolein in both water and triolein-water system along 255 the z-axis was monitored as seen in Fig. 4. At equilibrium, the obtained water and 256 triolein densities were 1000 and $926 \mathrm{~kg} / \mathrm{m}^{3}$, which represents the experiment data of 998 $257 \mathrm{~kg} / \mathrm{m}^{3}$ and $890 \mathrm{~kg} / \mathrm{m}^{3}$, respectively at $300 \mathrm{~K}$.

\subsection{Location of oleuropein in triolein-water system}

259 Figure3.b plots the density of the components in the simulation system along the z-axis. 260 Oleuropein exhibits two peaks $(6.99 \mathrm{~nm}$ and $13.58 \mathrm{~nm})$, indicating that oleuropein is 261 located on both sides of the triolein-water interfaces. Our observations are in line with 262 the corresponding experimental conclusions, that oleuropein exhibits interfacial activity 263 at the triglyceride-water interface (Souilem et al., 2014). Oleuropein caused a significant 264 decrease of the interfacial tension at the triglyceride-water interface and showed an 265 emulsifying ability to stabilize $\mathrm{O} / \mathrm{W}$ droplets using microchannel emulsification 266 (Souilem et al., 2014).

\section{3.3. Conformational analysis}

268 To gain further insight into the details of the environmental effects, the torsions 269 distribution in each environment was analyzed. Considering the large number of 
conformers generated from the MD simulations, the distribution of each torsion that was

271 previously defined in Fig.2.a, was determined. It can be easily seen from Fig.4 that the 272 resulting distribution plots for the major torsions are different in vacuum, water or 273 triolein-water systems except torsion 7 , reflecting the flexibility of oleuropein structure 274 in different surrounding microenvironments. The highest preferential locations of the 275 studied torsions are identified in Fig.4 as $\mathrm{T}_{\mathrm{nt}}^{\text {Sys }}$ (nt: number of torsion; Sys (system 276 (Vacuum (VAC), Water (WAT), Triolein-water (TRI-WAT)). Analyzing torsion1, two 277 dominant populations with maxima $\left(\mathrm{T}_{1}{ }^{\mathrm{VAC} 1}, \mathrm{~T}_{1}{ }^{\mathrm{VAC} 2}\right)=\left(\mathrm{T}_{1}{ }^{\mathrm{WAT} 1}, \mathrm{~T}_{1}{ }^{\mathrm{WAT} 2}\right)=\left(\mathrm{T}_{1}{ }^{\text {TRI-WAT1 }}\right.$, $\left.278 \mathrm{~T}_{1}{ }^{\mathrm{TRI}-\mathrm{WAT} 2}\right)=\left(-90^{\circ}, 92^{\circ}\right)$ were observed in all vacuum, water and triolein-water systems 279 The similar observed distribution plots of $T_{1}$ were characterized by a similar amplitude 280 of the maxima $\left(-90^{\circ}\right)$ at around $18 \%$ indicating a low solvent effect on the torsion 1 .

281 Torsion 2 exhibited different distribution plots in the studied systems. In vacuum, $\mathrm{T}_{2}$ 282 was more frequently located at $\mathrm{T}_{2}{ }^{\mathrm{VACl}}=176^{\circ}$ with an amplitude of $13.5 \%$ but it could 283 also be located at $\mathrm{T}_{2}{ }^{\mathrm{VAC} 2}=-65^{\circ}$ and $\mathrm{T}_{2}{ }^{\mathrm{VAC} 3}=71^{\circ}$ with lower amplitudes of $3.7 \%$ and $2847.5 \%$, respectively. Conversely, in water, $\mathrm{T}_{2}$ is located at two symmetric maxima $285\left(\mathrm{~T}_{2}{ }^{\mathrm{WAT} 1}, \mathrm{~T}_{2}{ }^{\mathrm{WAT} 2}\right)=\left(-65^{\circ},+65^{\circ}\right)$ having an amplitude of $12.9 \%$. We e also notice a 286 possible location $\mathrm{T}_{2}{ }^{\mathrm{WAT} 3}$ at $176^{\circ}$ but with lower observed amplitude at around $5.8 \%$.

287 In triolein-water, torsion 2 has the same preferential location as in vacuum. $\mathrm{T}_{2}^{\mathrm{VAC1}}=$ $288 \mathrm{~T}_{2}{ }^{\mathrm{TRI}-\mathrm{WAT} 1}=176^{\circ}$, but presents a higher amplitude of $22 \%$. The different preferential 289 angle of torsion 2 between the water and triolein-water systems suggests a staggered 290 conformation of the bond (CBD-CBC) and (CBB-OBA) might contribute to oleuropein 291 partitioning into the hydrophobic triolein phase. Analyzing torsion 3, two symmetric 292 dominant states were observed. In vacuum, the states have ranges of $\left[-138^{\circ},-34^{\circ}\right]$ and $293\left[27^{\circ}, 143^{\circ}\right]$, with maxima $\mathrm{T}_{3}{ }^{\mathrm{VACl}}$ and $\mathrm{T}_{3}{ }^{\mathrm{VAC} 2}$ of $-75^{\circ}$ and $82^{\circ}$. In water and in 
294 triolein-water systems, the ranges of the states are shifted to $\left[-176^{\circ},-74^{\circ}\right]$ and $\left[67^{\circ}\right.$, $295178^{\circ}$ ]. Two symmetric preferential torsional angle $\mathrm{T}_{3}{ }^{\mathrm{WAT} 1}=-139^{\circ}$ and $\mathrm{T}_{3}{ }^{\mathrm{WAT} 2}=153^{\circ}$ with 296 a similar occurrence of $10.2 \%$ were observed in water. In triolein-water, a larger 297 expansion of torsion 3 was observed leading to the staggered conformation of bond $298(\mathrm{CBC}-\mathrm{CBB})$ and $(\mathrm{OBA}-\mathrm{CAZ})$ at $\mathrm{T}_{1}{ }^{\mathrm{TRI}-\mathrm{WAT} 1}=-179^{\circ}$. The distribution plots of torsion 4 299 showed a pronounced solvent effect on its motion. In fact, in vacuum, torsion 4 is more 300 frequently at a staggered conformation $\mathrm{T}_{4}{ }^{\mathrm{VACl}}$ a $-179^{\circ}$ with high amplitude of $26 \%$. 301 However, in water or triolein-water, $\mathrm{T}_{4}$ varies among $-177^{\circ},-74^{\circ}$ and $82^{\circ}$. The maximum $302 \mathrm{~T}_{4}$ observed in water was $\mathrm{T}_{4}{ }^{\mathrm{WATl}}=-74^{\circ}$ and the highest observed location in 303 triolein-water was $\mathrm{T}_{4}{ }^{\text {TRI-WAT1 }}$ at $-179^{\circ}$. Analyzing torsion 5 , two parabolic dominant 304 states having the ranges of $\left[-152^{\circ},-65^{\circ}\right]$ and $\left[15^{\circ}, 126^{\circ}\right]$ described the populations of 305 torsion 5 in vacuum with a preference for $\mathrm{T}_{5}{ }^{\mathrm{VAC} 1}=80^{\circ}$ having an amplitude of $9.5 \%$. In 306 water and triolein-water systems, torsion 5 has more flexibility, explained by the border 307 distribution plots while keeping the same preferential angle at $\mathrm{T}_{5}{ }^{\mathrm{WAT} 1}=\mathrm{T}_{5}{ }^{\mathrm{TRI}-\mathrm{WAT} 1}=80^{\circ}$. 308 Torsion $\mathrm{T}_{6}$ exhibits two dominant states in vacuum, with the highest preference at $309 \mathrm{~T}_{6}{ }^{\mathrm{VACl}}=-67^{\circ}$. In water and triolein-water systems, different distribution plots of $\mathrm{T}_{6}$ were 310 observed, indicating the presence of intermolecular interactions in the presence of water 311 or triolein. In water, $\mathrm{T}_{6}$ has the highest population at $\mathrm{T}_{6}{ }^{\mathrm{WAT} 1}=74^{\circ}$ but in the 312 triolein-water system, it was observed to be distributed at two angles $\mathrm{T}_{6}{ }^{\text {TRI-WAT1 }}=74^{\circ}$ 313 and $\mathrm{T}_{6}{ }^{\mathrm{TRI}-\mathrm{WAT} 2}=164^{\circ}$ having a similar amplitude of around $17 \%$.

314 Torsion angle $\mathrm{T}_{7}$ linking the glucoside moiety to the oleuropein aglycone showed a 315 single and sharp preferential peak at around $-80^{\circ}$ in all systems, indicating its high 316 intramolecular rigidity through the simulation periods. In the triolein-water system with 317 different starting orientations of the oleuropein, the distribution plots were reproduced 
at the two interfaces for torsions $T_{2}, T_{3}, T_{4}$ and $T_{7}$. However, small deviations were obtained for torsions $T_{1}$ and $T_{5}$ which could explain the high flexibility of these torsions in a biphasic system.

321 Summarizing the above results, we noticed that torsions $T_{2}, T_{3}$ and $T_{4}$ showed the 322 highest microenvironmental dependence since they exhibited different distribution plots 323 and preferential angles depending on the system. In addition, the carboxyl ester group 324 linking hydroxytyrosol and elenolic acid is considered to be the key flexible region in 325 the oleuropein molecule.

\subsection{Flexibility of oleuropein}

\subsubsection{Conformation of oleuropein in vacuum}

329 The distribution of torsional angles in vacuum (Fig.4) showed that the preferential 330 torsions $\left(\mathrm{T}_{5}{ }^{\mathrm{VACl}}\right.$ and $\left.\mathrm{T}_{6}{ }^{\mathrm{VACl}}\right)$ are acute $\left(81^{\circ}\right.$ and $\left.69^{\circ}\right)$, suggesting that oleuropein folds 331 into a closed molecule. The distance $\left(\mathrm{d}_{\text {glu-hyd }}\right)$ during simulation was monitored for further investigation and distributions of $d_{\text {glu-hyd }}$ were illustrated in Fig. 5. The average distance $\mathrm{d}_{\text {glu-hyd }}$ was relatively stable at $0.6 \mathrm{~nm}$ with small fluctuations during the simulations. This confirms the close proximity of hydroxytyrosol to glucose and is consistent with the fact that all conformers have a tight structure (Fig6.a). Nevertheless, $\mathrm{d}_{\text {glu-hyd }}$ exceeds $0.32 \mathrm{~nm}$, indicating that there is no intramolecular interaction between

337 the atoms forming the hydroxytyrosol and glucose moieties. The corresponding $d_{\text {glu-hyd }}$ 338 values of the highest observed conformers $\mathrm{C}_{\mathrm{vac}}{ }^{1}, \mathrm{C}_{\mathrm{vac}}{ }^{2,} \mathrm{C}_{\mathrm{vac}}{ }^{3}$ and $\mathrm{C}_{\mathrm{vac}}{ }^{4}$ were $0.589 \mathrm{~nm}$, 339 $0.641 \mathrm{~nm}, 0.594 \mathrm{~nm}$ and $0.645 \mathrm{~nm}$, respectively. 


\subsubsection{Conformation of oleuropein in water}

342 Water highly influences on the torsion angle distribution (Fig.4), which would be 343 reflected in the conformational preference of oleuropein. The shift of $\mathrm{T}_{3}\left(\mathrm{~T}_{3}{ }^{\mathrm{VAC} 1} ; \mathrm{T}_{3} \mathrm{VAC} 2\right)$ 344 from $\left(-79^{\circ},+79^{\circ}\right)$ to the maxima $\left(\mathrm{T}_{3}{ }^{\mathrm{WAT} 1} ; \mathrm{T}_{3}{ }^{\mathrm{WAT} 2}\right)\left(-139^{\circ}, 154^{\circ}\right)$ in water may cause the 345 glucose moiety to be located further from the phenolic group of hydroxytyrosol in water. 346 Figure 5.b depicts the distributions of $d_{\text {glu-hyd }}$ in the water system. The $d_{\text {glu-hyd }}$ distance 347 varied from 0.4 to $1.4 \mathrm{~nm}$ with an average distance of $0.8 \mathrm{~nm}$. The high frequency of the 348 distance variation might reflect a fast dynamics of oleuropein between the tight and 349 open forms. The torsions analyses were in agreement with the distance analysis since 350 oleuropein preferential conformers (Fig.6) presented a large difference in their structural 351 properties, varying from a close like $U$ form $\left(\mathrm{C}_{\mathrm{wat}}{ }^{1}\right)$ having a $\mathrm{d}_{\text {glu_hyd }}$ distance of 0.570 $352 \mathrm{~nm}$, and a semi-open form $\left(\mathrm{C}_{\mathrm{wat}}{ }^{3}\right)$ having a $\mathrm{d}_{\mathrm{glu}-\mathrm{hyd}}$ of $1.074 \mathrm{~nm}$ to an opened form like 353 the conformer, $\mathrm{C}_{\text {wat }}{ }^{4}$ with a $\mathrm{d}_{\text {glu-hyd }}$ of $1.214 \mathrm{~nm} \cdot$ The high flexibility of oleuropein is 354 expected to be potentially influenced by the presence of water, which may interact with 355 a number of $\mathrm{OH}$ groups throughout the oleuropein molecule by forming hydrogen 356 bonds.

357

358 3.4.3. Conformation of oleuropein in the triolein-water system

359 The preferential torsions $\left(\mathrm{T}_{2}{ }^{\text {TRI-WAT }}, \mathrm{T}_{3}{ }^{\text {TRI-WAT }}\right.$ and $\mathrm{T}_{4}{ }^{\text {TRI-WAT }}$ ) linking the hydroxyphenyl 360 and elenolic acid moieties have the same rotation around $\left(-180^{\circ}, 180^{\circ}\right)$ (Fig.4) 361 suggesting that oleuropein folds into an open molecule. Similarly to the water system, $362 \mathrm{~d}_{\text {glu-hyd }}$ ranged from 0.4 to $1.4 \mathrm{~nm}$ with an average distance of $0.84 \mathrm{~nm}$. However, less 363 fluctuation of the distance was observed as compared to its motion in the water system. 364 The structures of the highest probable conformers in the triolein-water system are 
represented in Fig. 6c. The top 10 highest probable conformers for oleuropein followed an opened conformation. However, there are several notable differences in the orientations of the glucose and hydroxyphenyl moieties in a set of opened conformations. $\mathrm{d}_{\text {glu-hyd }}$ of the different selected conformers varies between $1.008 \mathrm{~nm}$ for $\mathrm{C}_{\text {tri_wat }}{ }^{8}$ and $1.377 \mathrm{~nm}$ for $\mathrm{C}_{\text {tri_wat }}{ }^{6}$. The greatest $\mathrm{d}_{\text {glu-hyd }}$ distance forces the glucose, elenolic acid and hydroxytyrosol to be approximately aligned and indicates high repulsive forces inside the molecule.

\subsection{Energy determination of preferential conformers}

Since the major conformations of oleuropein were different for each system, the required energy to adopt each conformation was predicted (Table 1). In vacuum, $\mathrm{S}_{\mathrm{vac}}{ }^{1}$ was the most stable conformation since it has the lowest energy. The energy difference between the lowest energy conformer and the highest energy conformer in vacuum is about $9.73 \mathrm{kcal} / \mathrm{mol}$, which strongly indicates that oleuropein can easily adopt these different conformations. Similarly, based on the energy values of the highest probable conformers, the stability of oleuropein in water appears in this order: $\mathrm{C}_{\mathrm{wat}}{ }^{4}>\mathrm{C}_{\mathrm{wat}}{ }^{2}>\mathrm{C}_{\mathrm{wat}}{ }^{1}>\mathrm{C}_{\mathrm{wat}}{ }^{3}$, which means that the most stable conformation in water has an open form where the glucose, elenolic acid and hydroxytyrosol are approximately aligned. However, the energy barrier between the closed conformer and the open form is small, about $1.92 \mathrm{kcal} / \mathrm{mol}$, confirming that oleuropein can easily convert between the two forms.

In the triolein-water system, oleuropein showed flexibility lower than that in the water system since all of the conformational states have a relatively open form (Fig.6.c). All of the conformers are geometrically symmetric; they differ in the orientation of their 
389 glucose and hydroxyphenyl moieties. The conformer $\left(\mathrm{C}_{\text {tri-wat }}{ }^{9}\right)$ presented the lowest 390 energy, which reflects its higher stability in the triolein-water system when adopting this 391 conformation state. $\mathrm{C}_{\text {tri-wat }}{ }^{9}$ is characterized by a $\mathrm{d}_{\text {glu-hyd }}$ of $1.142 \mathrm{~nm}$, which is lower than 392 the distance of $\mathrm{S}_{\text {tri-wat }}{ }^{6}$, so repulsive forces are minimized in this structure. The energy 393 barrier of $14.39 \mathrm{kcal} / \mathrm{mol}$ makes the difficult to change from the conformer $\mathrm{C}_{\text {tri-wat }}{ }^{9}$ to $394 \mathrm{C}_{\text {tri-wat }}^{2}$.

395 When comparing the energy difference between all the systems, we found that the 396 energy barrier of oleuropein to convert from the tight form representing the lowest 397 energy in vacuum to the lowest energy form in a water system is around $5.24 \mathrm{kcal} / \mathrm{mol}$. 398 However, the energy barrier between the lowest energy conformer in water and 399 triolein-water systems is around $19.14 \mathrm{kcal} / \mathrm{mol}$. This energy barrier is required for 400 oleuropein to cross the system. The $\mathrm{O} / \mathrm{W}$ interfacial activity of oleuropein might 401 originate from specific hydrogen bonding and hydrophobic interactions at the 402 triolein-water interface that is expected to greatly contribute to its stabilization.

3.6. Radial distribution function analysis: Interaction of oleuropein with the 404 surrounding environment

405 Radial distribution function analyses (RDF) for the water solution in water and in 406 triolein-water systems were calculated based on the MD simulations. We focus on the 407 oxygen atom, i.e $\mathrm{O}$ (oleuropein)- $\mathrm{O}$ (water), where $\mathrm{O}$ (oleuropein) refers to an oxygen 408 atom belonging to oleuropein and $\mathrm{O}$ (water) refers to the surrounding water. Based on 409 these results, hydrogen bonding is expected when the cut-off of the well-defined peak at 410 a distance below $3.5 \AA$. Examples of radial distribution from different oxygen atoms in 411 the oleuropein molecule are presented in Fig.7. The oxygen atoms present in the glucose 
412 moiety reveal the existence of hydrogen bonding between the hydroxyl groups of 413 oleuropein $(\mathrm{O} 2, \mathrm{O} 3, \mathrm{O} 4$ and $\mathrm{O} 6)$, and water molecules both in bulk water and 414 triolein/water systems. For instance, for O6, there were sharp peaks at around $3.2 \AA$ in 415 water and $3 \AA$ in triolein/water. However, the presence of water molecules was steadier 416 in water (9.97 water molecules) than in the triolein/water system (2.52 water 417 molecules). A similar solvation effect was caused by the triolein/water system when 418 analyzing the oxygen atoms OBK and OBL belonging to the hydroxytyrosol moiety of 419 oleuropein (Fig.7.b). These atoms have stronger hydrogen bonding in a water system.

420 Analyzing the RDF plots for oxygen atoms(OAR, OAW, OAV, OBE, and OBA), 421 belonging to the elenolic acid moiety, one or more water molecules was found around 422 VCDB HY67UJP/MQWSOAR, OAV and OBE in the water system (Table 2), 423 confirming the existence of hydrogen bonding in these regions. However, these curves 424 were significantly less intense in the triolein/water system, suggesting that an effective 425 desolvation happened beyond the first shell of the corresponding oxygen atoms (Table 426 2).For OAR, the shell completely disappears, which leads to the inaccessibility of water 427 molecules to the elenolic acid moiety.

428 Summarizing the above results, when we compare the RDF plots in water and in 429 triolein/water systems, the main differences are found for the $\mathrm{O}$ (elenolic acid)-O water 430 interactions and to lower extents in the glucose and hydroxytyrosol moieties. These 431 results confirm the $\mathrm{O} / \mathrm{W}$ interfacial activity of oleuropein which was assumed based on 432 the lowering of solvation of the main oxygen atoms in oleuropein. The absence of 433 hydrogen bonding in the elenolic acid region suggests its partitioning into the triolein 434 phase due to hydrophobic interactions. 


\subsection{Link between conformation and $\mathrm{O} / \mathrm{W}$ interfacial activity of oleuropein}

437 We have confirmed the O/W interfacial location of oleuropein by the use of three sets of

438 simulations S1, S2 and S3 starting either from a parallel or a perpendicular initial 439 orientation of oleuropein to the triolein-water interface (Fig.2b). The energy barrier

440 between the lowest energy conformers in vacuum and water is around $5.24 \mathrm{kcal} / \mathrm{mol}$.

441 However, the energy barrier between the lowest energy conformers in vacuum and 442 triolein-water is around $13.90 \mathrm{Kcal} / \mathrm{mol}$. This high energy barrier deems more stability 443 of oleuropein in the triolein-water system and leads to a specific adsorption 444 conformation adopted at the interface. RDF analysis suggested that the conformational 445 change of oleuropein significantly affected its hydrophilic and hydrophobic properties. 446 The semi-open conformation of oleuropein in water had total water coordination as high 447 as 42 molecules while the open conformation of oleuropein at the triolein-water 448 interface had a total water coordination 11 molecules. The oleuropein at the 449 triolein-water interface had the adsorption enthalpy of -36.21 to $-34.83 \mathrm{kcal} / \mathrm{mol}$. This 450 suggests that the open conformation of oleuropein greatly increases its hydrophobicity, 451 and facilitates its binding at the triolein-water interface. Torsional analysis together with 452 the $\mathrm{d}_{\mathrm{glu}-\mathrm{hyd}}$ variation during equilibrium revealed an open conformation of oleuropein at 453 the triolein-water interface but did not inform about the orientation of the molecule 454 toward the interface. The interfacial activity of oleuropein is assumed to be due the 455 presence of hydrophilic and hydrophobic moieties. Our previous assumption 456 approached oleuropein to the glucose ester surfactant where the hydrophilic moiety is 457 formed by glucose while the hydrophobic tail is formed by oleuropein aglycone 458 (Souilem et al. 2014). However, RDF analysis showed the presence of hydrogen 
459 bonding related to OBK and OBL oxygens, which implies the partitioning of some 460 branches of hydroxytyrosol into the water phase. Based on the above results, we 461 propose that oleuropein adopted a horizontal open conformation parallel to the 462 triolein-water interface following the illustration in Fig. 9, in which the hydrophobic 463 part is formed by elenolic acid whereas the hydrophilic part is formed by the glucose 464 moiety and some branches of hydroxytyrosol. The strong hydrophobic intermolecular 465 interactions explained the lower conformational dynamics of oleuropein at the 466 triolein-water interface as compared to the water system, where the molecule could 467 easly convert between the open and close forms (Fig.7b). The proposed orientation of 468 oleuropein at the triolein-water interface may explain the large areas of occupied 469 oleuropein per interface determined experimentally using the Gibbs theory (Souilem et 470 al. 2014). To gain information about the nature of adsorption of oleuropein at 471 triolein-water interface, we have calculated the enthalpy of its adsorption for the three 472 sets of simulations S1, S2 and S3 mentioned above. The obtained enthalpies values 473 converged to similar ranges of values, $-36.21 \pm 4.26,-34.83 \pm 3.66$ and $-34.99 \pm 6.47$ $474 \mathrm{kcal} / \mathrm{mol}$ for the configurations in $\mathrm{S} 1, \mathrm{~S} 2$ and $\mathrm{S} 3$, respectively. $\Delta \mathrm{H}<0$ implies that 475 adsorption occurs spontaneously and it is exothermic, a result in agreement with the 476 higher obtained energies of conformers in triolein-water system (Table 1). Although the 477 enthalpy of adsorption is a fundamental reflection of the interaction between the 478 adsorbate and an interface, it could be determined experimentally based on interfacial 479 tension measurements (Krassimir and Kralchevsky.2012). The interfacial tension of the 480 pure triolein-water system has been measured by Meyers et al. (2012) using a pendent 481 drop tensiometer to be $32.1 \mathrm{mN} / \mathrm{m}$, a value which is close to the interfacial tension of the 482 pure soybean oil-water system $(31.5 \mathrm{mN} / \mathrm{m})$ (Goankar, 1989). Therefore, we roughly 
estimated the free enthalpy of adsorption of oleuropein based on interfacial tension 484 results of oleuropein containing soybean oil-water systems (Fig. 9). Interfacial tension 485 data at different temperatures were plotted in accordance with Eq (1) (Fig. 9a). The 486 internal free energy of oleuropein adsorption $(\Delta \mathrm{G})$ was derived from the intercepts of 487 linear regressions of these plots (Fig. 9b). $\Delta \mathrm{G}$ was around -35.81 and $-41.73 \mathrm{KJ} / \mathrm{mol}$ for $48825^{\circ} \mathrm{Cand} 45^{\circ} \mathrm{C}$, respectively. The obtained experimental $\Delta \mathrm{G}$ values were plotted against 489 temperature in order to extract $\Delta \mathrm{H}$ according to the fit with Eq (2) (Fig. 9c). The obtained 490 experimental enthalpy was around $-31.86 \mathrm{KJ} / \mathrm{mol}(-7.61 \mathrm{Kcal} / \mathrm{mol})$. Experimental 491 calculations confirmed that oleuropein adsorption at $\mathrm{O} / \mathrm{W}$ interface is an enthalpy driven 492 mechanism. The higher enthalpy of adsorption obtained experimentally is expected since 493 many oleuropein molecules are aggregated at the interface and the enthalpy of adsorption 494 is dependent on the adsorbate concentration (Anwar-ul-Haq et al. 2013).

495 Complementary MD simulations of the adsorption of many oleuropein molecules at 496 triolein-water interface would offer a better comparaison with the experimental 497 adsorption kinetics of oleuropein. Moreover, we aim to extend our studies to look at the 498 effect of $\mathrm{pH}$, ionic strength and temperature on the interfacial activity of oleuropein, and 499 ultimately hope to lead a better understanding of the factors that control its stability and 500 delivery when incorporated in multiphasic systems

501

\section{4. Conclusions}

503 The present study provides accessible conformational models for oleuropein in different 504 environments (vacuum, water and triolein-water systems). The data indicated that 505 oleuropein undergoes extensive conformational changes depending on the 506 microenvironment. The nature of the molecule dynamics whether it is in open, 
507

508

509

510

511

512

513

514

515

516

517 food emulsions.

518 Acknowledgment

519 The authors are grateful to the Science and Technology Research Partnership for 520 Sustainable Development (SATREPS) Project, financially supported by JICA and JST, 521 Japan.

522

523

524

525 Fig.2. (a) Atom numbering of oleuropein and definition of the seven torsional angles.(b)

526 Sets of simulations of oleuropein in triolein-water system.

527 Fig.3. Density profiles of the different systems. (a) water; (b) oleuropein-water-triolein 528 system.

semi-open or tight form could be easily identified from torsion analyses and from the distance between the glucose and hydroxyphenyl moieties of oleuropein. Water mediated hydrogen bonding was greatly involved in stabilizing the three-dimensional conformation of oleuropein. The absence of hydrogen bonding with water molecule in the elenolic acid moiety of oleuropein might be the key towards its stabilization at the triolein-water interface, mainly due to hydrophobic interactions. This MD study confirmed our previous experiment results regarding the surface activity of oleuropein at the triglyceride-water interface (Souilem et al. 2014) and provides us with a full understanding of the amphiphilic character of oleuropein at the molecular level. The surface activity of oleuropein might influence the preparation, stability and delivery of

\section{Figures and tables captions}

Fig. 1. Chemical structure of (a) oleuropein and (b) triolein. 
529 Fig.4. Dihedral angles distributions in vacuum (left side), water (center) and 530 triolein-water (right side) systems.

531 Fig.5. Distributions of distance between hydroxytyrosol and glucose moieties ( $\left.\mathrm{d}_{\text {glu-hyd }}\right)$ 532 throughout the trajectories during equilibrium: (a) vacuum; (b) water; (c) triolein-water 533 system.

534 Fig.6. Highest probable conformers of oleuropein in (a) vacuum, (b) water and (c) 535 triolein-water systems. (d) Alignment of oleuropein in vacuum (left side), water (center), and 536 triolein-water (right side) system

537 Fig.7. Oleuropein-water radial distribution functions:(a) water ; (b) triolein-water

538 Fig.8. Partitioning of oleuropein at the triolein-water interface

539 Fig.9. Interfacial tension data and related adsorption enthalpies of oleuropein at soybean 540 oil-water interface.

541 Table 1.Dihedral angles and energy information for the highest probable conformations 542 of oleuropein in vacuum, water and triolein-water systems.

543 Table 2.Radial Distribution Function (RDF) analyses results.(a) water system.(b) 544 triolein-water system.

545

\section{$546 \quad$ References}

547 Andreadou, I., Iliodromitis, E. K., Mikros, E., Constantinou, M., Agalias, A., Magiatis, $548 \quad$ P., Skaltsouni, A.L., Kamber, E., Kakoulidou, A.T., \&Kremastinos, D. T. (2006). 549 The olive constituent oleuropein exhibits anti-ischemic, antioxidative, and 550 hypolipidemic effects in anesthetized rabbits. The Journal of Nutrition, 136(8), $551 \quad 2213-2219$.

552 Anwar-ul-Haq, A.S., Khurshid, A., \& Salma, B. (2013). Surface tension, surface excess 553 concentration, enthalpy and entropy of surface formation of aqueous salt solutions. 
Colloids and Surfaces A: Physicochemical and Engineering Aspects,417, 183190.

Bisignano, G., Tomaino, A, Lo Cascio, R., Crisafi, G., Uccella, N., \& Saija, A. (1999). On the invitro antimicrobial activity of oleuropein and hydroxytyrosol. The Journal of Pharmacy and Pharmacology, 51(8), 971-974.

Bulotta, S., Corradino, R., Celano, M., Maiuolo, J., D’Agostino, M., Oliverio, M., Procopio, A., Filetti, S., \&Russo, D. (2013). Antioxidant and antigrowth action of peracetylated oleuropein in thyroid cancer cells. Journal of Molecular Endocrinology, 51(1), 181-189.

Capasso, R., Evidente, A., Visca, C., Gianfreda, L., Maremonti, M., \& Greco, G. (1997). Production of glucose and bioactive aglycone by chemical and enzymatic hydrolysis of purified oleuropein fromOlea Europea. Applied Biochemistry and Biotechnology, 61(3), 365-377.

Caturla, N., Pérez-Fons, L., Estepa, A., \& Micol, V. (2005). Differential effects of oleuropein, a biophenol from Olea europaea, on anionic and zwiterionic phospholipid model membranes. Chemistry and Physics of Lipids, 137(1-2), 2-17.

Cicerale, S., Lucas, L., \& Keast, R. (2010). Biological activities of phenolic compounds present in virgin olive oil. International Journal of Molecular Sciences, 11(2), $458-79$.

Di Giovacchino, L., Costantini, N., Ferrante, M. L., \& Serraiocco, A. (2002). Influence of malaxation time of olive paste on oil extraction yields and chemical and organoleptic characteristics of virgin olive oil obtained by a centrifugal decanter at water saving. Grasas Y Aceites, 53(2), 179-186.

Di Mattia, C. D., Sacchetti, G., \& Pittia, P. (2011). Interfacial Behavior and Antioxidant Efficiency of Olive Phenolic Compounds in O/W Olive oil Emulsions as Affected by Surface Active Agent Type. Food Biophysics, 6(2), 295-302.

Ghahfarokhi, A. K., Dadashi, A., Daryasafar, A.,\&Moghadasi, J. (2015). Feasibility study of new natural leaf-derived surfactants on the IFT in an oil-aqueous system: 
584 Ghanbari, R., Anwar, F., Alkharfy, K. M., Gilani, A. H., \& Saari, N. (2012). Valuable 585 nutrients and functional bioactives in different parts of olive (Olea europaea L.)-A review. International Journal of Molecular Sciences, 13, 3291-3340.

587

588

589

590

591

592

593

594

595

596

597

598

599

600

601

602

603

604

605

606

607

608

609

610

Gikas, E., Bazoti, F. N., \& Tsarbopoulos, A. (2007). Conformation of oleuropein, the major bioactive compound of Olea europea. Journal of Molecular Structure: THEOCHEM, 821(1-3), 125-132.

Gaonkar, A. (1989). Interfacial tensions of vegetable oil/water systems: Effect of oil purification. Journal of the American Oil Chemists' Society, 66(8), 1090-1092.

Gutierrez-Rosales, F., Romero, M. P., Casanovas, M., Motilva, M. J., \& Mínguez-Mosquera, M. I. (2010). Metabolites involved in oleuropein accumulation and degradation in fruits of Olea europaea L.: Hojiblanca and Arbequina varieties. Journal of Agricultural and Food Chemistry, 58(24), 12924-12933.

Henneré, G., Prognon P., Brionc F.,\&Nicolis I. (2009).Molecular dynamics study of a phospholipid monolayer at awater/triglyceride interface: towards lipid emulsion modelling.Chemistry and Physics of Lipids, 157, 86-93.

Huang, P. L., Huang, P. L., \& Lee-Huang, S. (2010). Oleuropein and Related Compounds Reduce AtherosclerosisThe Open Conference Proceedings Journal, $1(1), 81-86$.

Kumari,R., Kumar,R., \&Lynn, A. (2014). g_mmpbsa-A GROMACS tool for high-throughput MM-PBSA calculations Journal of Chemical Information and Modeling, 54, 1951-1962.

Krassimir, D.D., \& Kralchevsky, P.A. (2012). The standard free energy of surfactant adsorption at air/water and oil/water interfaces: Theoretical vs. empirical approaches. Colloid Journal, 74, 172-185. 
611 Lee-Huang, S., Huang, P. L., Zhang, D., Lee, J. W., Bao, J., Sun, Y., Chang, Y. T Y., 612 Zhang, J.,\&Huang, P. L. (2007). Discovery of small-molecule HIV-1 fusion and 613 integrase inhibitors oleuropein and hydroxytyrosol: Part I. Integrase inhibition. 614 Biochemical and Biophysical Research Communications, 354(4), 872-878.

615 Leopoldini, M., Russo, N., \& Toscano, M. (2011). The molecular basis of working 616 mechanism of natural polyphenolic antioxidants. Food Chemistry, 125(2), $617 \quad 288-306$.

López-Miranda, J., Pérez-Jiménez, F., Ros, E., De Caterina, R., Badimón, L., Covas, M. 619 I., ... Yiannakouris, N. (2010). Olive oil and health: summary of the II 620 International Conference on Olive Oil and Health Consensus report, Jaén and 621 Córdoba (Spain) 2008. Nutrition, Metabolism, and Cardiovascular Diseases: $622 \quad$ NMCD, 20(4), 284-94.

Meyers, N., Wang, L., \& Small, D. (2012). Apolipoprotein C-I binds more strongly to phospholipid/triolein/ water than triolein/water interfaces: a possible model for inhibiting cholesterol ester transfer protein activity and triacylglycerol-rich lipoprotein uptake. Biochemistry, 51(6), 1238-1248.

627

Omar, S. H. (2010). Oleuropein in olive and its pharmacological effects. Scientia 628

Souilem, S., Kobayashi, I., Neves, M. A., Jlaiel, L., Isoda, H., Sayadi, S., \& Nakajima, 630

631

632 M. (2014). Interfacial characteristics and microchannel emulsification of oleuropein-containing triglyceride oil-water systems. Food Research

633 Tieleman, P., MacCallum, J., Ash, W., Kandt, C., Xu, Z., \& Monticelli, L (2006). 634 Menbrane protein simulations with a united-atom lipid and all-atom protein model: 635 lipid-protein interactions, side chain transfer free energies and model proteins. 636 Journal of Physics: Condensed Matter, 18,28. 
637 Uccella, N. (2000). Olive biophenols: biomolecular characterization, distribution 638 andphytoalexin histochemical localization in the drupes. Trends in Food Science \& 639 Technology, 11(9-10), 315-327.

640 Internet

641 http://wcm.ucalgary.ca/tieleman (Access date: December 17, 2014).

642

643

644 
(a)

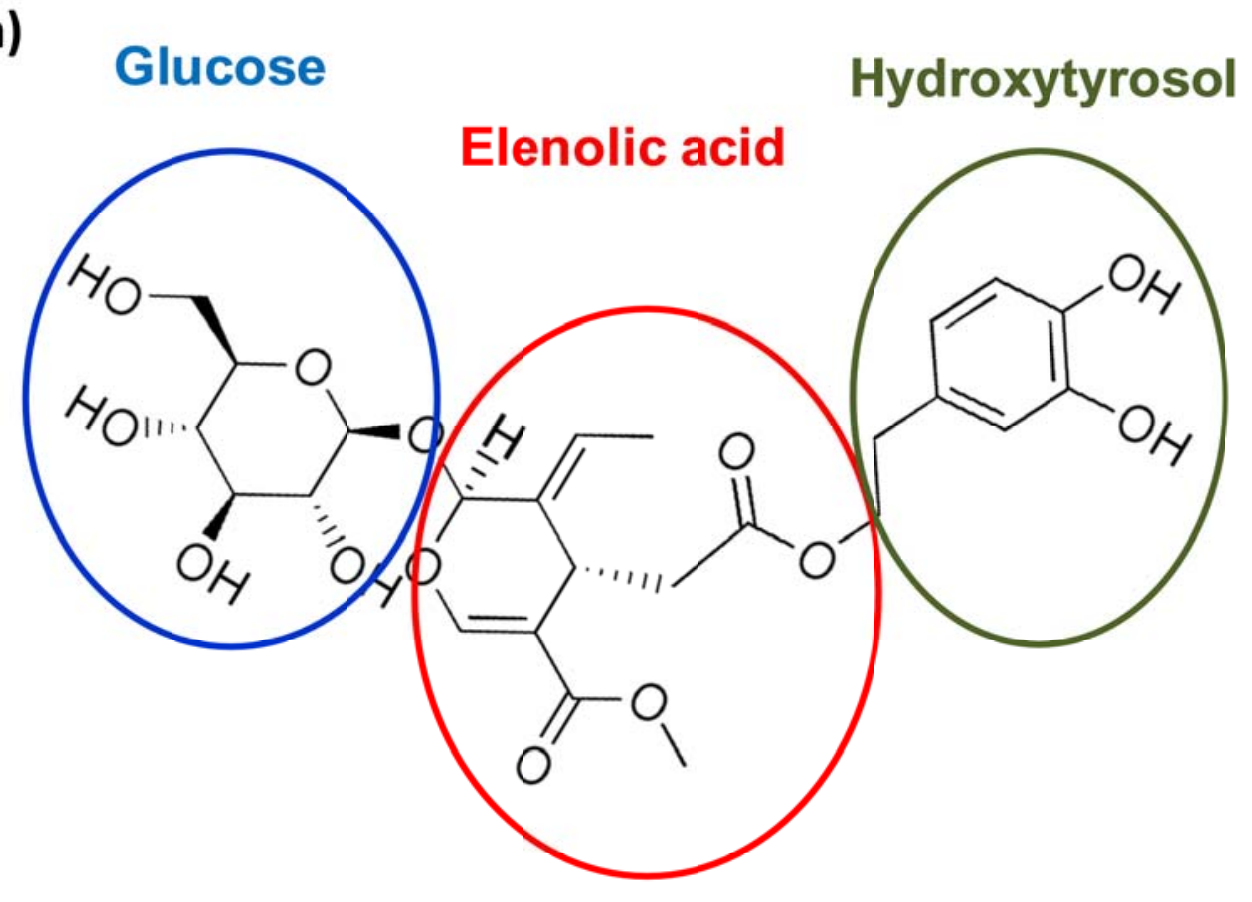

(b)

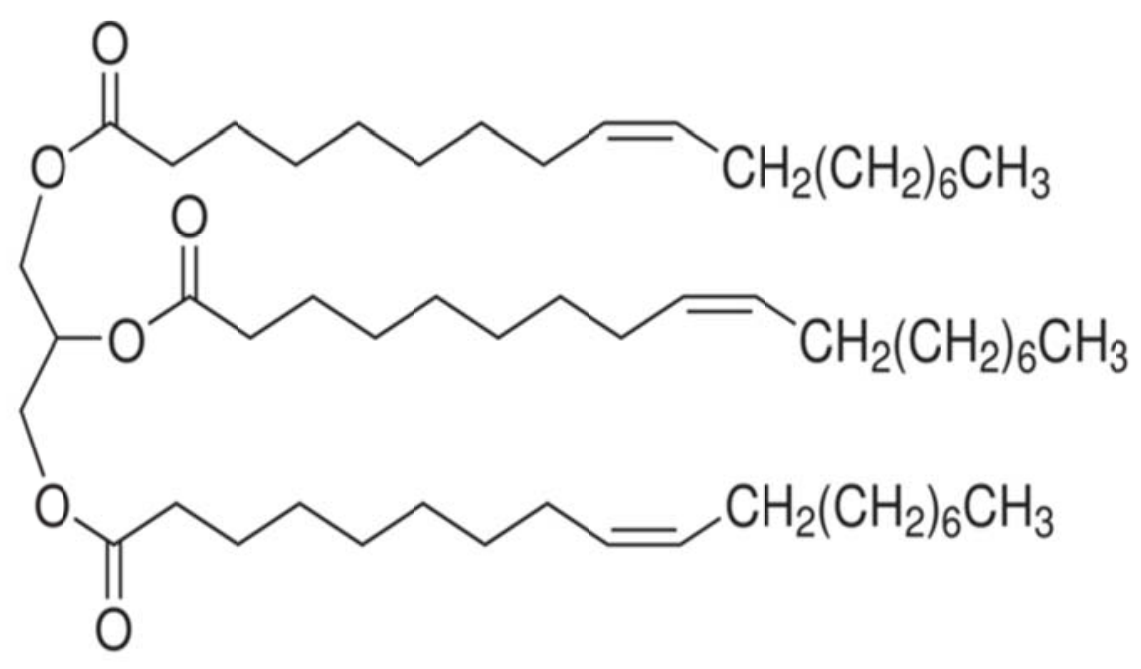

Fig 1. 
(a)

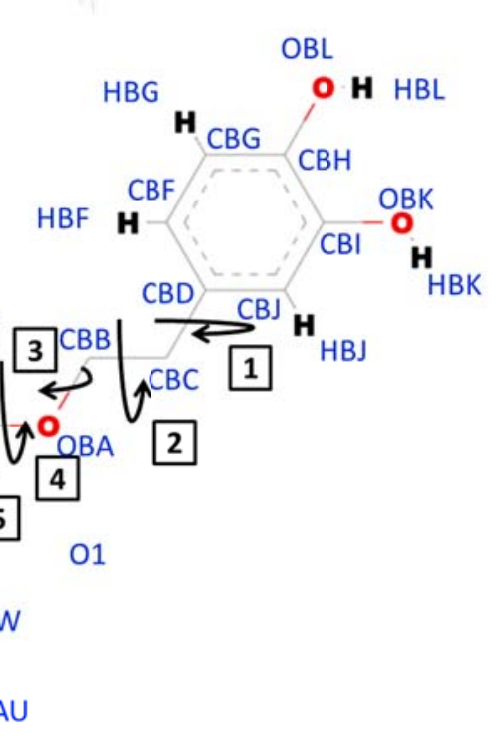

(b) $\mathrm{S1}$

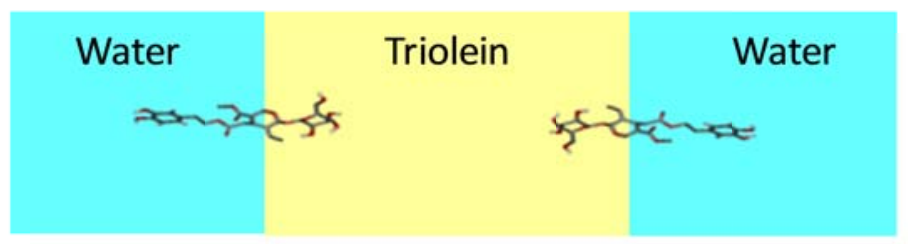

S2

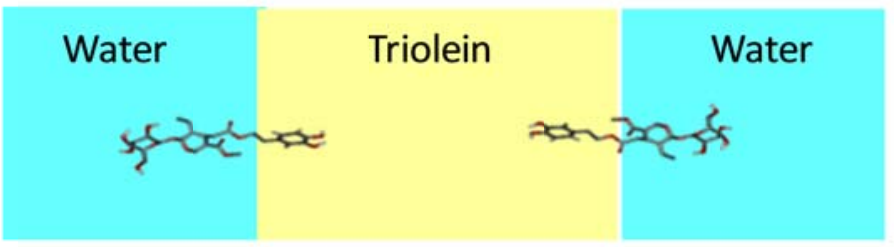

S3

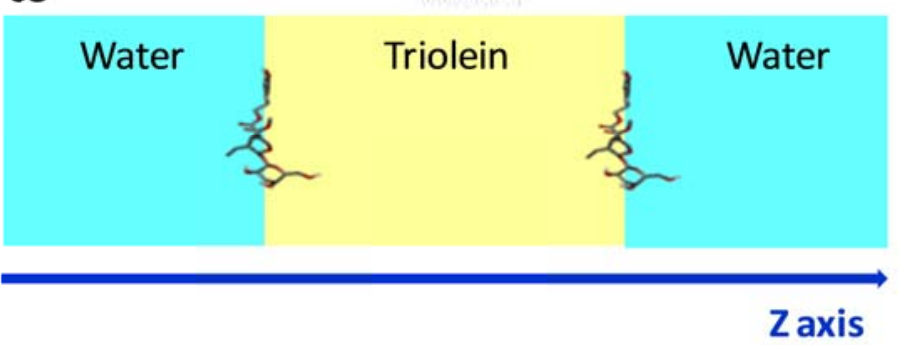

Fig 2. 
(a)

(b)
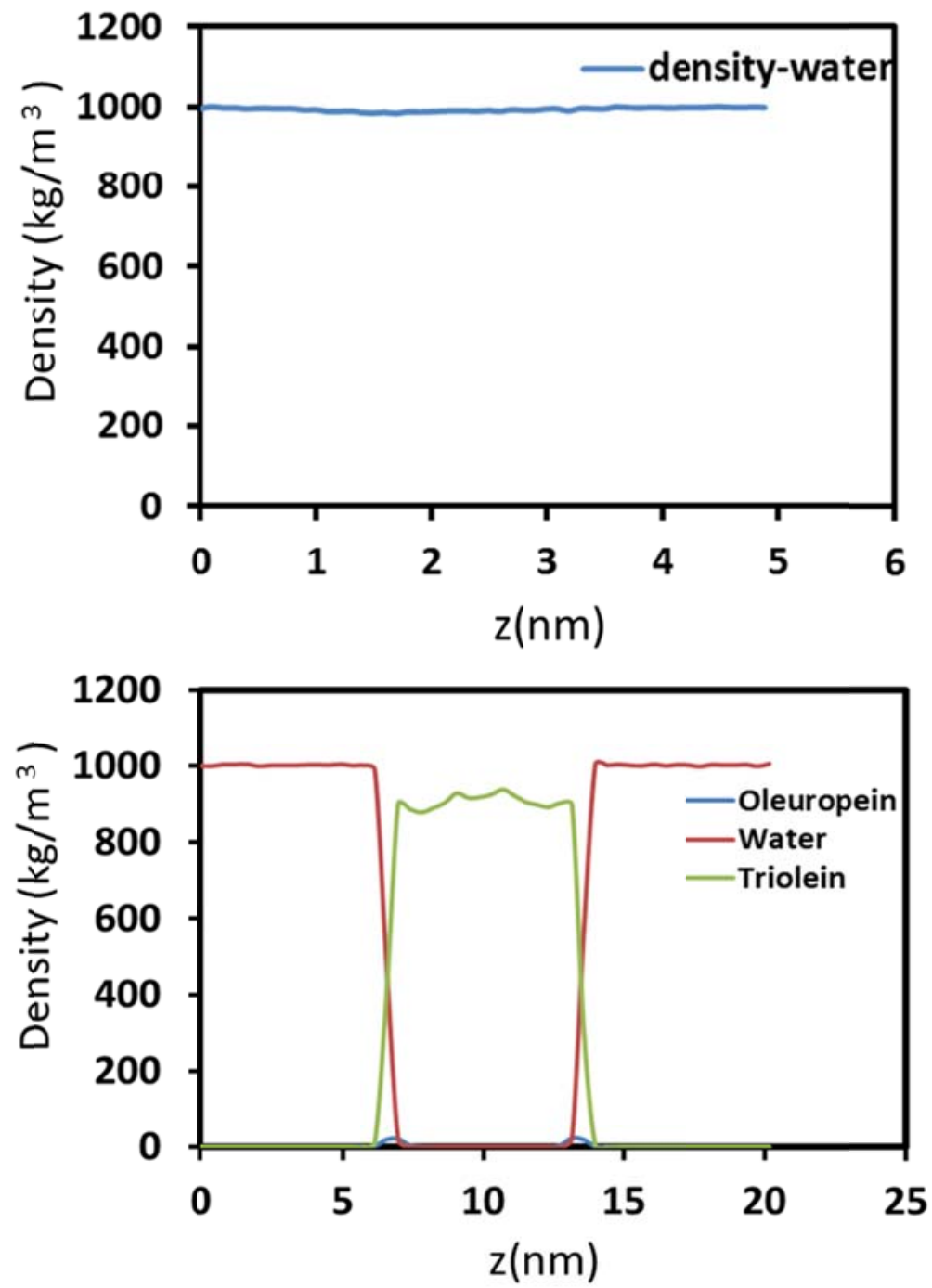

Fig 3.

Souilem et al. 


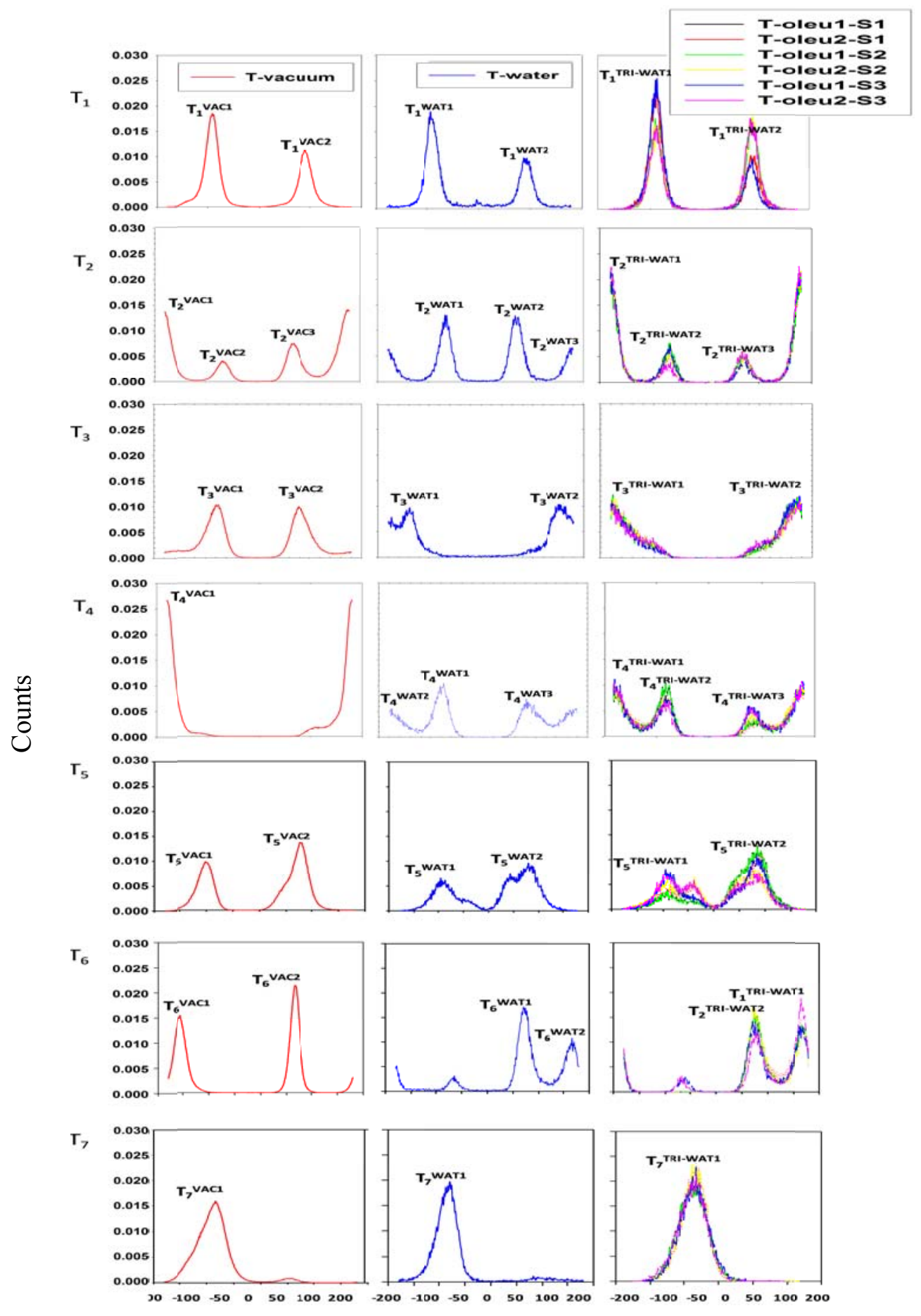

Fig 4.

Souilem et al. 

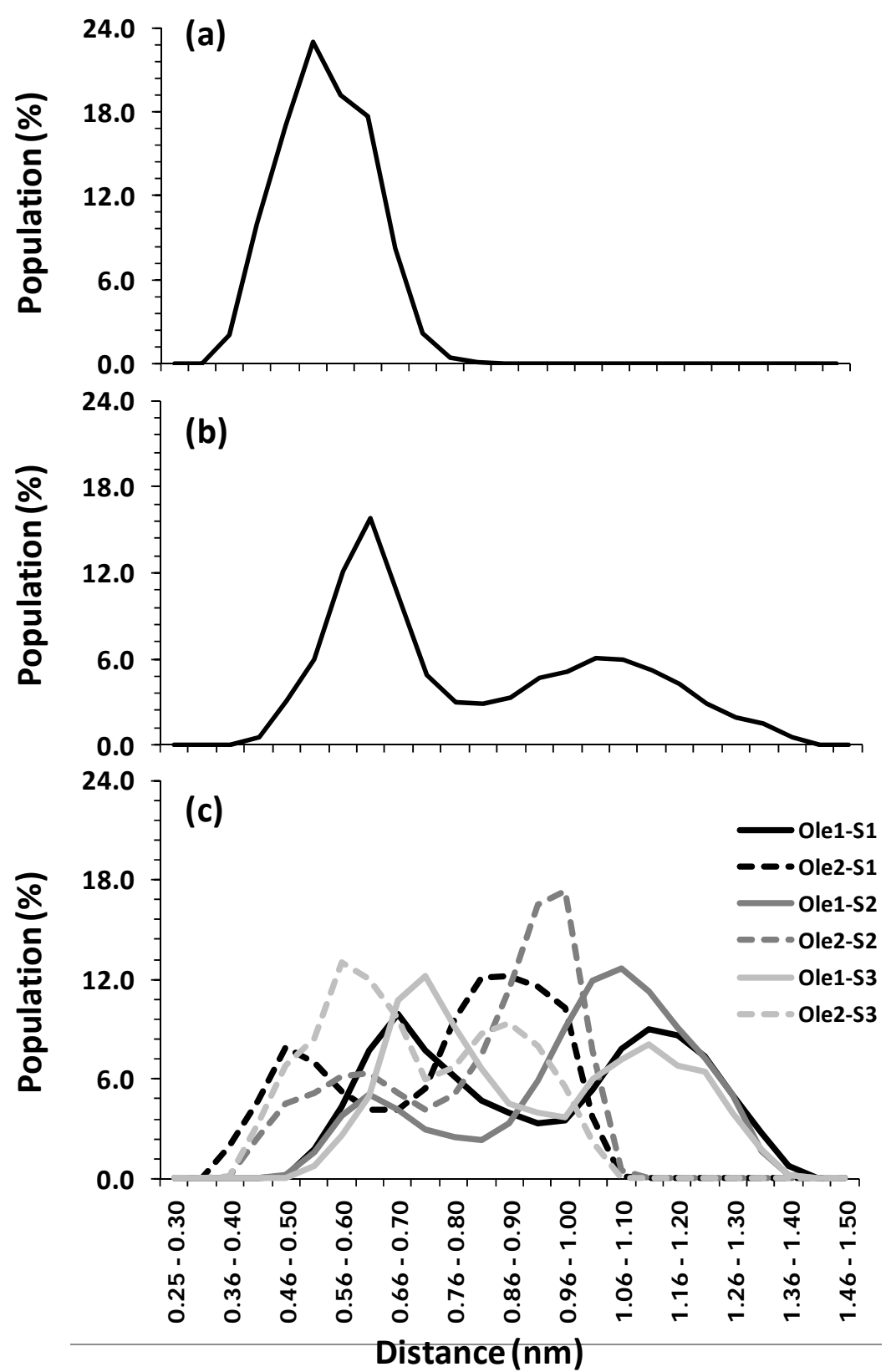

Fig 5.

Souilem et al. 
(a)
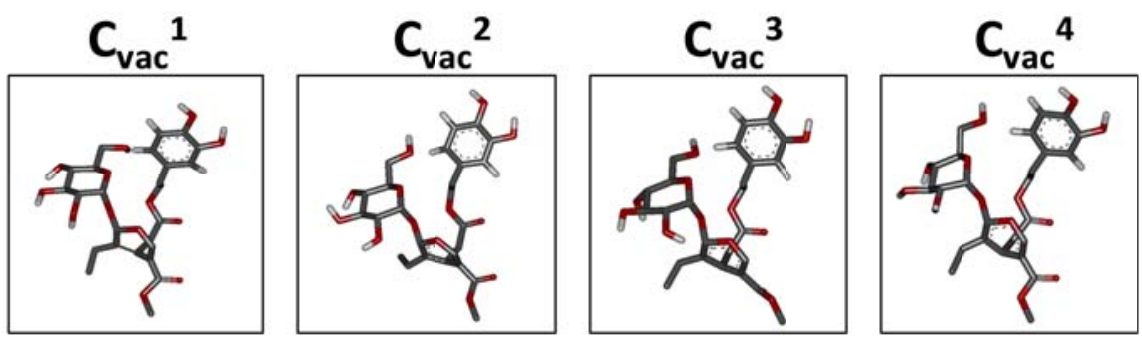

(b)
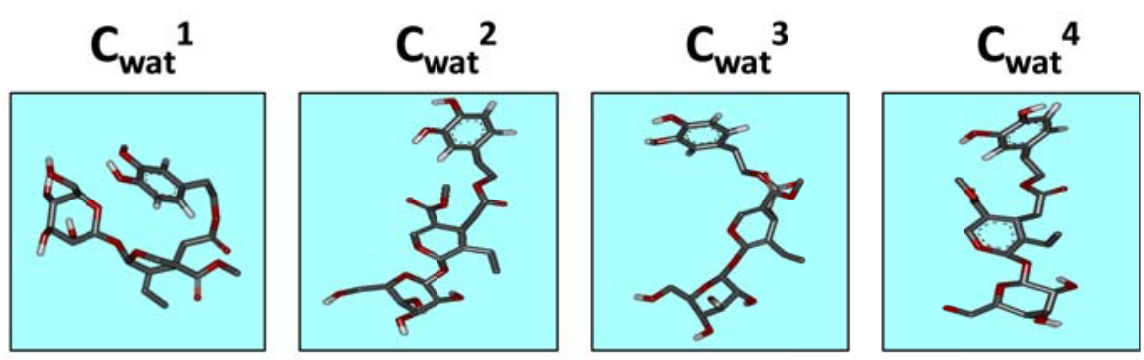

(c)
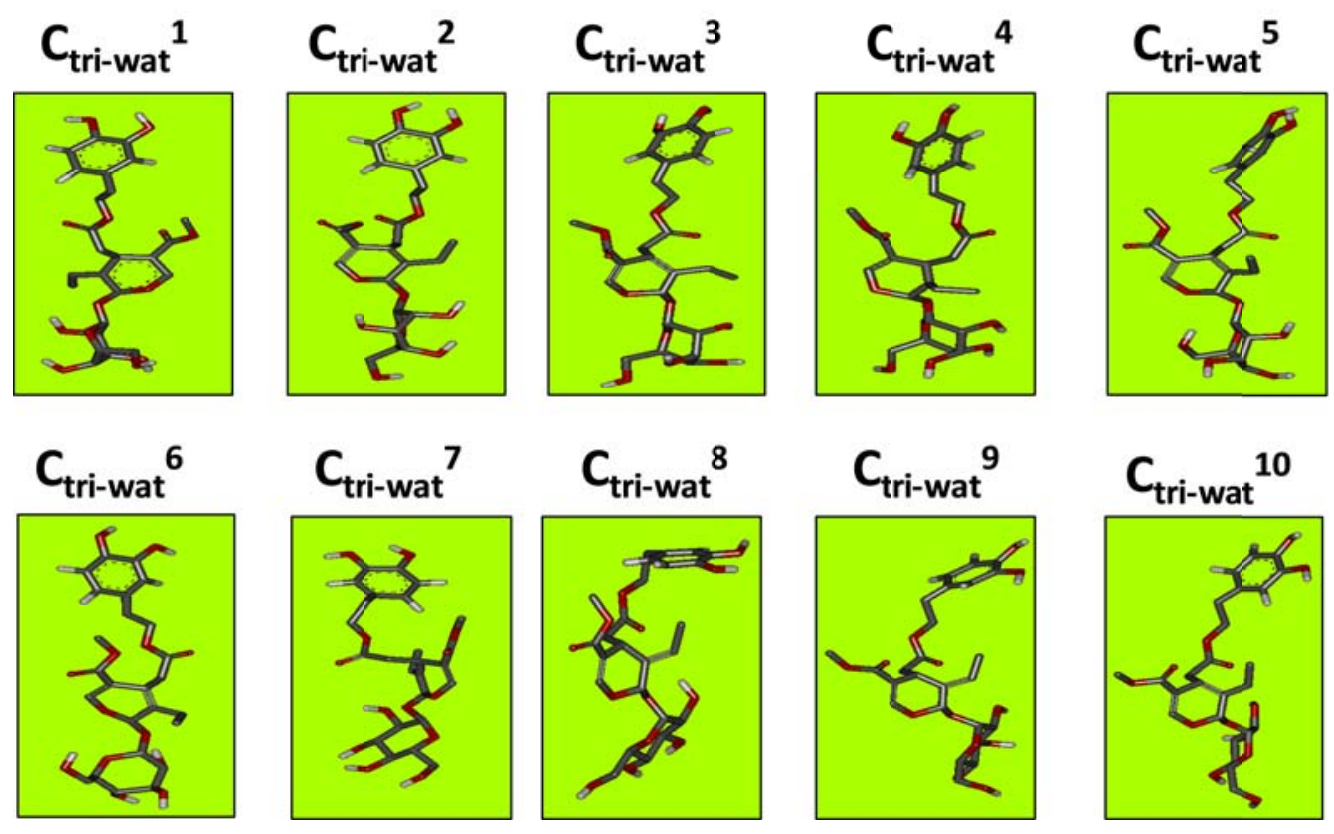

(d) Alignment of oleuropein in vacuum (left side), water (center), and triolein-water (right side) system 


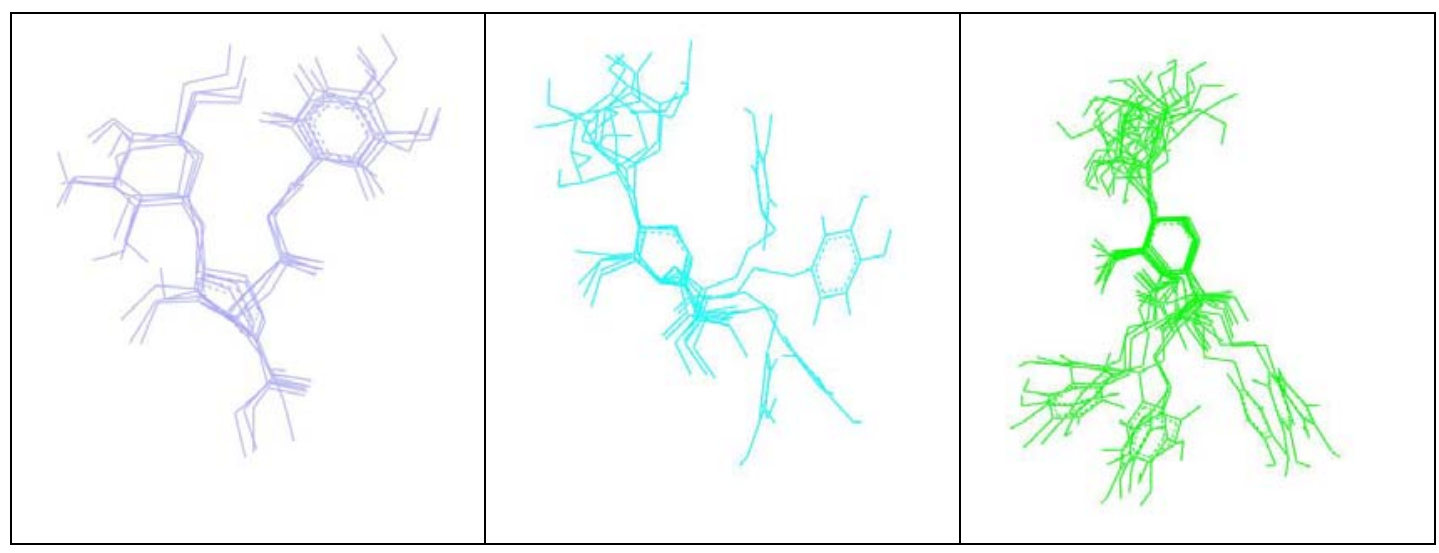

Fig6.

Souilem et al. 
Table 1. Dihedral angles and energy information of the highest probable conformations of oleuropein in vacuum, water and triolein-water systems

\begin{tabular}{|c|c|c|c|c|c|c|c|c|}
\hline \multirow[b]{2}{*}{ Conformer } & \multirow[b]{2}{*}{$\begin{array}{c}\text { Energy-difference } \\
\quad(\mathbf{k c a l} / \mathbf{m o l})\end{array}$} & \multicolumn{7}{|c|}{ Torsions in vacuum system } \\
\hline & & $\mathrm{T}_{1}$ & $\mathrm{~T}_{2}$ & $\mathrm{~T}_{3}$ & $\mathrm{~T}_{4}$ & $\mathrm{~T}_{5}$ & $\mathrm{~T}_{6}$ & $\mathrm{~T}_{7}$ \\
\hline$S_{\mathrm{vac}}{ }^{1}$ & 0 & -83.33 & -170.08 & -74.83 & -179.85 & 85.61 & -60.78 & -67.77 \\
\hline$S_{\mathrm{vac}^{2}}$ & 6.48 & -69.01 & 162.38 & -60.66 & -173.69 & 97.89 & -60.70 & -75.44 \\
\hline$S_{\mathrm{vac}}{ }^{3}$ & 9.73 & -83.45 & -173.96 & -86.80 & 179.41 & 76.96 & -57.70 & -98.32 \\
\hline \multirow[t]{2}{*}{$\mathrm{S}_{\mathrm{vac}}{ }^{4}$} & 5.95 & -89.29 & 175.23 & -89.24 & 177.89 & 85.34 & -50.49 & -101.83 \\
\hline & & \multicolumn{7}{|c|}{ Torsions in water system } \\
\hline Conformer & Energy & $\mathrm{T}_{1}$ & $\mathrm{~T}_{2}$ & $\mathrm{~T}_{3}$ & $\mathrm{~T}_{4}$ & $\mathrm{~T}_{5}$ & $\mathrm{~T}_{6}$ & $\mathrm{~T}_{7}$ \\
\hline$S_{\mathrm{wat}}{ }^{1}$ & 1.92 & -99.16 & -72.81 & 152.04 & -79.03 & 64.74 & 57.75 & -64.69 \\
\hline$S_{w a t}^{2}$ & 0.02 & -86.88 & 73.17 & 155.35 & -89.63 & 59.19 & 84.18 & -69.19 \\
\hline$S_{w a t}{ }^{3}$ & 6.13 & -89.02 & -67.98 & -148.36 & -81.94 & 72.02 & 64.77 & -81.47 \\
\hline \multirow[t]{2}{*}{$S_{\text {wat }}{ }^{4}$} & 0 & -95.11 & 71.28 & -146.72 & -116.80 & 93.95 & 79.03 & -96.61 \\
\hline & & \multicolumn{7}{|c|}{ Torsions in triolein-water system } \\
\hline Conformer & Energy & $\mathrm{T}_{1}$ & $\mathrm{~T}_{2}$ & $\mathrm{~T}_{3}$ & $\mathrm{~T}_{4}$ & $\mathrm{~T}_{5}$ & $\mathrm{~T}_{6}$ & $\mathrm{~T}_{7}$ \\
\hline$S_{\text {tri-wat }} 1$ & 19.83 & -98.44 & -177.94 & 159.70 & -175.13 & 81.61 & 101.80 & -77.89 \\
\hline$S_{\text {tri-wat }}^{2}$ & 14.39 & -82.90 & -155.65 & -170.59 & -174.99 & 107.64 & 154.81 & -90.02 \\
\hline $\mathbf{S}_{\text {tri-wat }}{ }^{3}$ & 21.37 & 93.88 & -148.72 & -149.98 & 151.00 & 84.64 & 146.54 & -74.49 \\
\hline$S_{\text {tri-wat }}{ }^{4}$ & 18.99 & -81.45 & 174.15 & -159.95 & 166.91 & 81.74 & 84.05 & -95.73 \\
\hline$S_{\text {tri-wat }}{ }^{5}$ & 18.99 & 93.38 & 169.62 & -171.04 & 159.61 & 75.92 & 138.24 & -74.41 \\
\hline$S_{\text {tri-wat }}{ }^{6}$ & 17.26 & 97.56 & 163.46 & 170.30 & -179.87 & 71.55 & 80.82 & -116.54 \\
\hline$S_{\text {tri-wat }}{ }^{7}$ & 02.27 & -92.41 & 164.83 & -172.70 & -167.37 & 75.23 & 74.54 & -84.58 \\
\hline$S_{\text {tri-wat }}{ }^{8}$ & 06.07 & -84.98 & -179.34 & -175.61 & 164.82 & 74.61 & 74.33 & -94.41 \\
\hline$S_{\text {tri-wat }}{ }^{9}$ & 0 & -86.31 & 164.99 & -169.93 & 166.32 & 100.99 & 162.19 & -125.13 \\
\hline$S_{\text {tri-wat }}{ }^{10}$ & 04.12 & -83.57 & 170.95 & 178.64 & 177.44 & 94.82 & 162.84 & -125.10 \\
\hline
\end{tabular}

Souilem et al. 
Table 2: RDF results in water and in triolein-water systems

\begin{tabular}{cccccc}
\hline \multirow{2}{*}{ Moiety } & & \multicolumn{2}{c}{ Water } & \multicolumn{2}{c}{ Triolein/water } \\
& Oxygen label & $\mathrm{r}(\AA)$ & $\mathrm{n}$ & $\mathrm{r}(\AA)$ & $\mathrm{n}$ (Owater) \\
& & & $($ Owater $)$ & & \\
\hline \multirow{3}{*}{ Glucose } & O2 & 3.04 & 2.11 & 3.00 & 0 \\
& O3 & 2.96 & 3.90 & 2.98 & 0.83 \\
& O4 & 3.10 & 2.93 & 3.16 & 1.88 \\
& O5 & 3.02 & 0.88 & $* *$ & $* *$ \\
& O6 & 3.20 & 9.97 & 3.04 & 2.52 \\
\hline \multirow{3}{*}{ Elenolic acid } & OAR & 3.46 & 5.55 & $*$ & $*$ \\
& OAV & 2.78 & 2.11 & 2.82 & 0.83 \\
& OAW & $* *$ & $* *$ & $* *$ & $* *$ \\
& OBE & 2.80 & 2.44 & 2.84 & 0.97 \\
& OBA & $* *$ & $* *$ & $* *$ & $* *$ \\
\hline \multirow{2}{*}{ Hydroxytyrosol } & OBK & 3.04 & 5.61 & 2.94 & 2.17 \\
& OBL & 2.96 & 6.12 & 2.92 & 2.04 \\
\hline
\end{tabular}

$*$ : no shell $\quad * *$ : $r>$ cut-off distance 
(a)
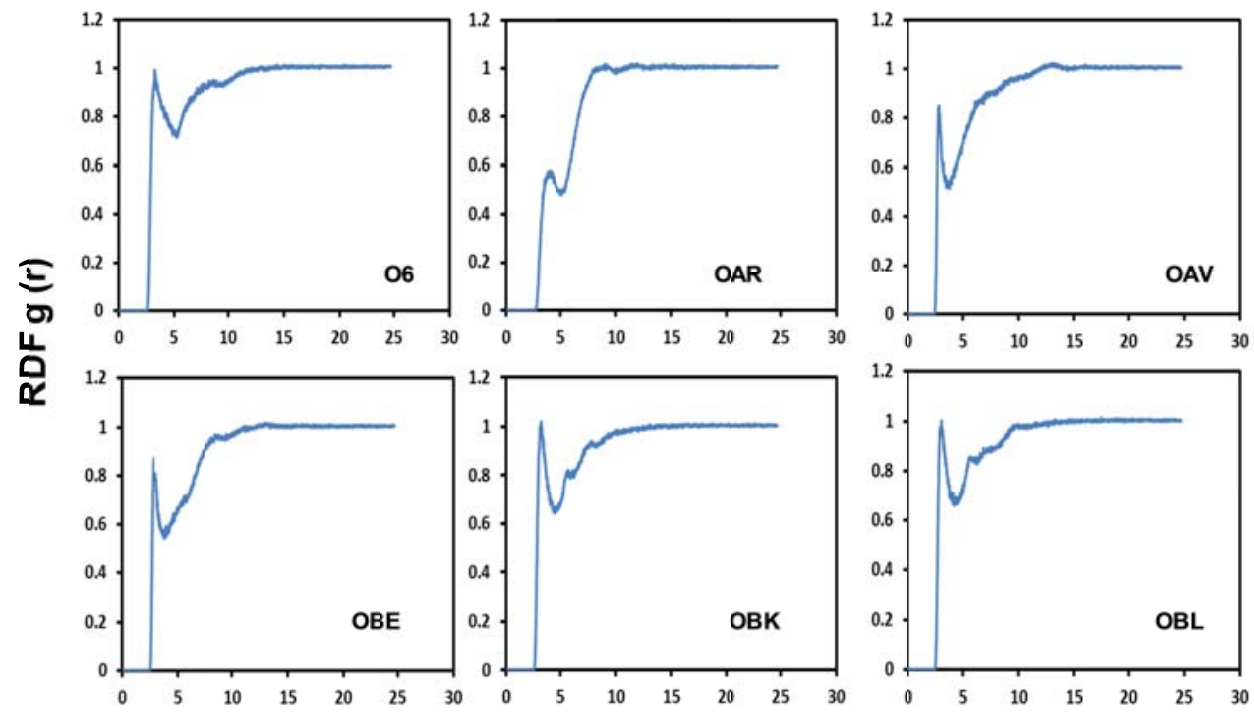

(b)
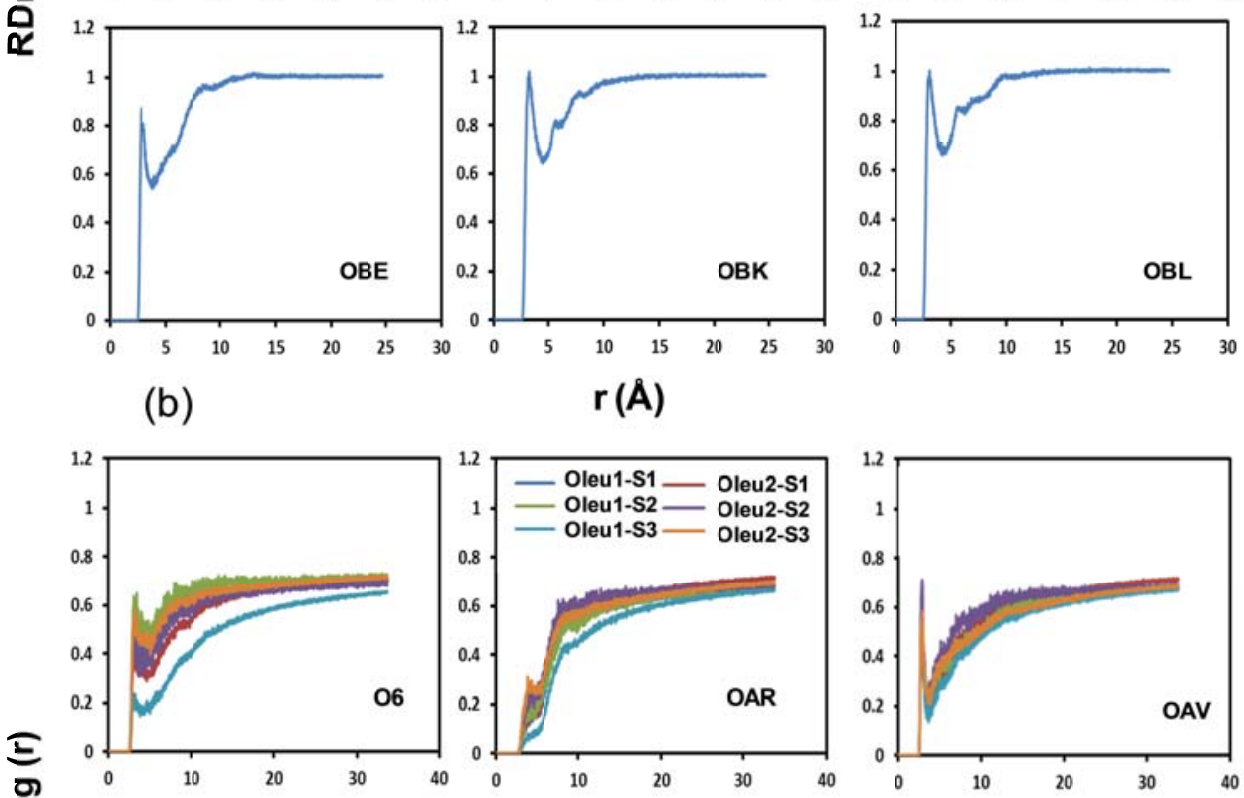

$r(A)$
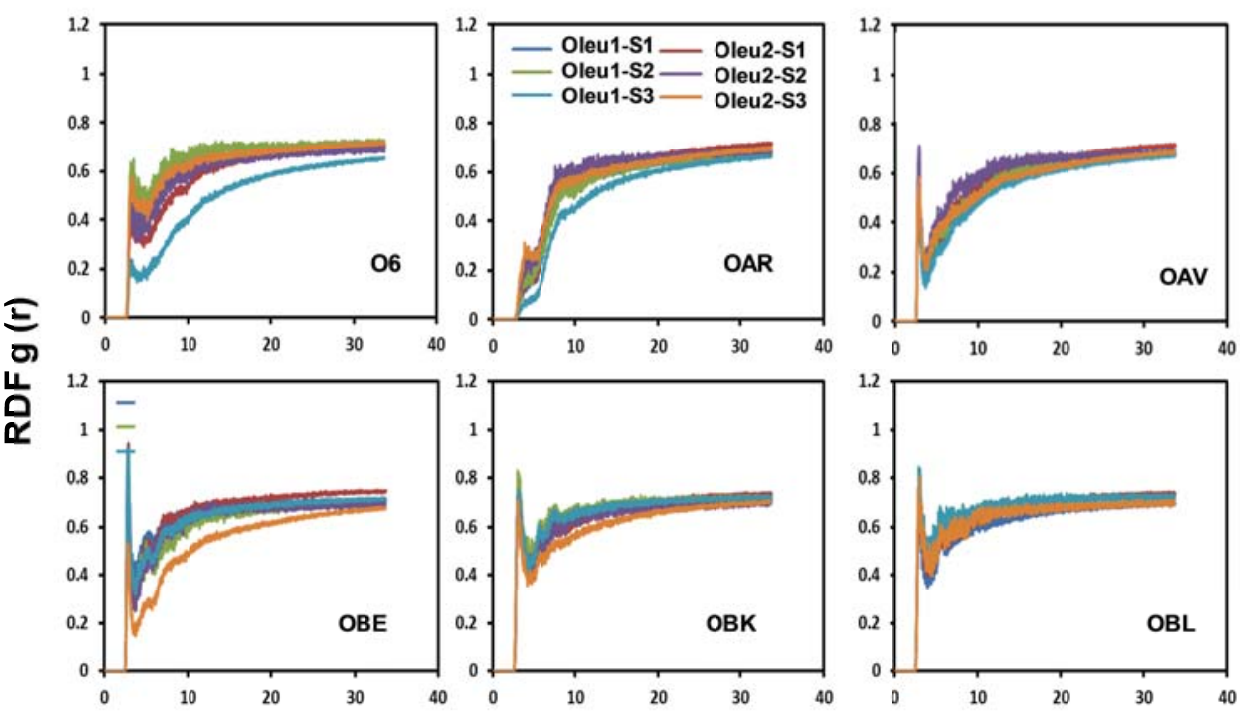

$r(\AA)$

Fig. 7

Souilem et al. 


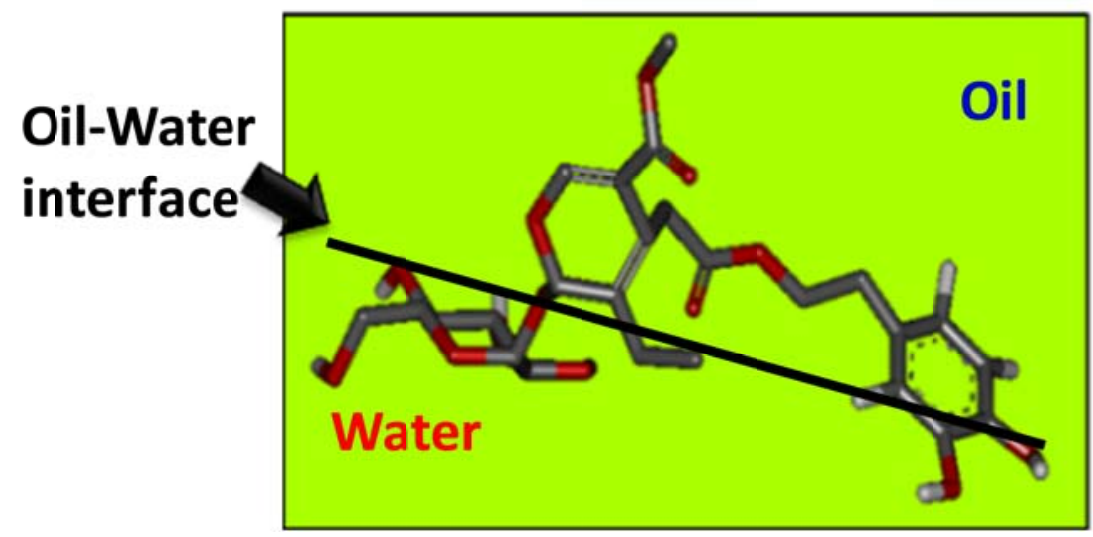

Fig. 8

Souilem et al. 
(a)

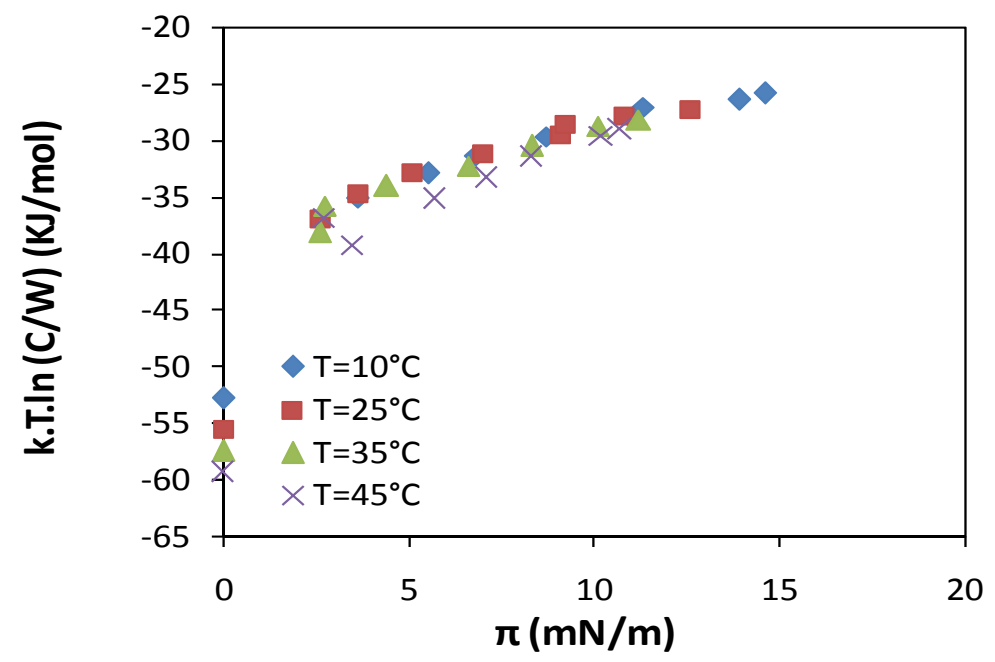

(b)

(c)
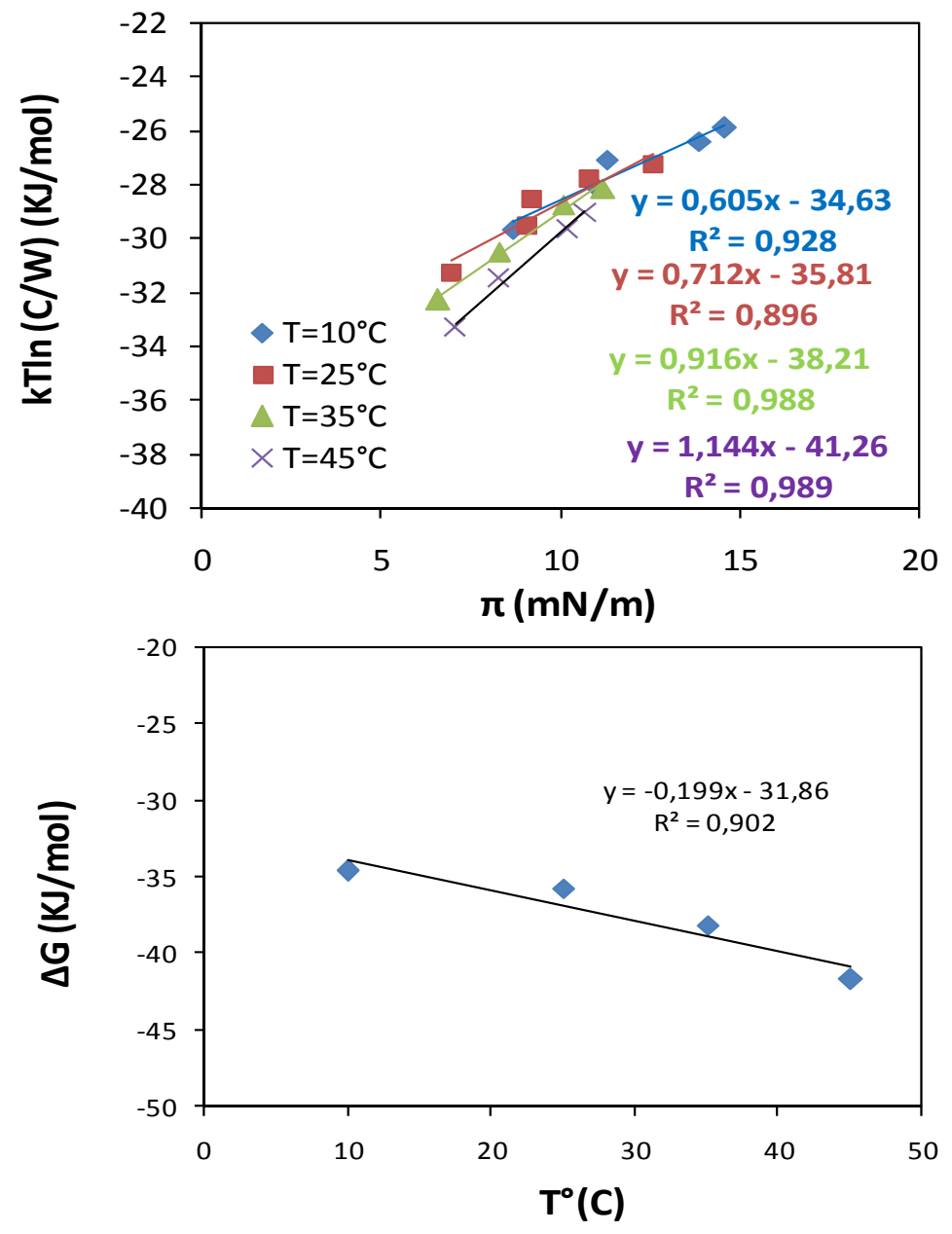

Fig. 9

Souilem et al. 